\title{
OBSERVATIONS OF COMETS
}

Throughout Part I of this volume the astronomical dates are used as employed before $\mathbf{1 9 2 5}$ 


\begin{tabular}{|c|c|c|c|c|c|c|c|c|c|c|}
\hline \multicolumn{11}{|c|}{ DANIEL (1907 d) } \\
\hline \multirow{2}{*}{ Date } & \multirow{2}{*}{ W. M. T. } & \multicolumn{2}{|c|}{ Apparent Place of Comet } & \multicolumn{2}{|c|}{ Comet-Star } & \multirow{2}{*}{ Comp. } & \multicolumn{2}{|c|}{$\log p \rho$} & \multicolumn{2}{|c|}{ Star to App. Place } \\
\hline & & $\alpha$ & $\delta$ & $\alpha$ & $\delta$ & & $\alpha$ & $\delta$ & $\alpha$ & $\delta$ \\
\hline $\begin{array}{l}1908 \\
\text { Feb. } \quad 3\end{array}$ & $\begin{array}{rrr}\mathrm{h} & \mathrm{m} & \mathrm{s} \\
17 & 20 & 43\end{array}$ & $\begin{array}{rrr}\mathrm{h} & \mathrm{m} & \mathrm{s} \\
15 & 8 & 41.90\end{array}$ & $\begin{array}{rr}\circ & \circ \\
-85 & 2.9\end{array}$ & $\stackrel{\mathrm{m}}{-2}$ 57. 06 & -311.1 & $\mathrm{t} 25,5$ & 9. $042 n$ & 0.810 & $\begin{array}{c}\mathrm{s} \\
-0.83\end{array}$ & $\begin{array}{l}\text { " } \\
+1.7\end{array}$ \\
\hline \multicolumn{11}{|c|}{ MOREHOUSE (1908 c) } \\
\hline $\begin{array}{r}1908 \\
\text { Sept. } 6 \\
12 \\
15 \\
22 \\
30\end{array}$ & $\mid \begin{array}{rrr}13 & 24 & 54 \\
8 & 29 & 12 \\
8 & 22 & 50 \\
8 & 54 & 52 \\
13 & 58 & 16\end{array}$ & $\begin{array}{rrr}3 & 6 & 16.16 \\
2 & 28 & 32.69 \\
1 & 57 & 37.63 \\
23 & 59 & 53.59 \\
21 & 20 & 43.52\end{array}$ & 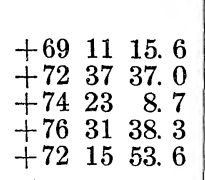 & 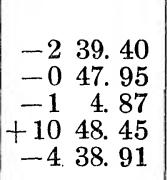 & $\begin{array}{r}+413.0 \\
+1242.7 \\
+237.4 \\
+1034.5 \\
+441.6\end{array}$ & $\begin{array}{r}\mathrm{t} 25,5 \\
\mathrm{t} 6,2 \\
\mathrm{t} 15,5 \\
\mathrm{t} 9,2 \\
\mathrm{t} 25,5\end{array}$ & $\begin{array}{l}9.912 n \\
0.180 n \\
0.230 n \\
0.140 n \\
0.167\end{array}$ & $\begin{array}{l}0.477 n \\
0.410 \\
0.138 \\
0.538 n \\
9.633\end{array}$ & $\begin{array}{l}+3.48 \\
+4.71 \\
+5.61 \\
+6.20 \\
+2.90\end{array}$ & $\begin{array}{l}-8.1 \\
-4.9 \\
-2.2 \\
+10.4 \\
+21.4\end{array}$ \\
\hline $\begin{array}{ll}\text { Oct. } & 15 \\
& 16\end{array}$ & $\begin{array}{rrr}8 & 54 & 6 \\
11 & 59 & 12\end{array}$ & $\begin{array}{llll}19 & 26 & 29 . & 27 \\
19 & 22 & 55 . & 51\end{array}$ & $\begin{array}{r}+494733.4 \\
+474751.1\end{array}$ & $\begin{array}{ll}-1 & 55.57 \\
-0 & 57.41\end{array}$ & $\begin{array}{r}-\quad 012.0 \\
+\quad 026.7\end{array}$ & $\begin{array}{l}\mathrm{t} 25,5 \\
\mathrm{t} 25,5\end{array}$ & $\begin{array}{l}\text { 9. } 708 \\
9.832\end{array}$ & $\begin{array}{l}8.908 n \\
0.611\end{array}$ & $\begin{array}{l}+0.60 \\
+0.56\end{array}$ & $\begin{array}{l}+22.1 \\
+21.7\end{array}$ \\
\hline 18 & $\begin{array}{lll}8 & 50 & 32\end{array}$ & $1917 \quad 47.41$ & +442948.1 & -128.35 & -428.4 & $\mathrm{t} 25,5$ & 9.693 & 9.932 & +0.54 & +21.0 \\
\hline 20 & 82530 & $\begin{array}{llll}19 & 13 & 13.49\end{array}$ & $+41 \quad 124.6$ & -055.06 & -451.2 & $\mathrm{t} 25,5$ & 9.648 & 0.037 & +0.53 & +20.0 \\
\hline Nov. $\begin{array}{r}5 \\
12 \\
15 \\
17\end{array}$ & $\begin{array}{lll}8 & 31 & 48 \\
7 & 44 & 48 \\
6 & 19 & 46 \\
7 & 29 & 38\end{array}$ & $\begin{array}{rrr}18 & 55 & 6.54 \\
18 & 52 & 26.56 \\
18 & 51 & 45.39 \\
18 & 51 & 23.19\end{array}$ & $\begin{array}{r}+1641 \\
+843 \\
+843 \\
+547 \\
+352.6 \\
+352\end{array}$ & $\begin{array}{rr}-0 & 29.20 \\
+1 & 4.25 \\
+1 & 48.36 \\
+0 & 16.81\end{array}$ & $\begin{array}{rrr}- & 6 & 3.1 \\
+ & 2 & 34.3 \\
- & 2 & 10.0 \\
- & 0 & 7.1\end{array}$ & $\begin{array}{l}\mathrm{t} 25,5 \\
\mathrm{t25}, 5 \\
\mathrm{t} 25,5 \\
\mathrm{t} 18,6\end{array}$ & $\begin{array}{l}9.649 \\
9.622 \\
9.524 \\
9.622\end{array}$ & $\begin{array}{l}0.659 \\
0.698 \\
0.699 \\
0.724\end{array}$ & $\begin{array}{l}+0.56 \\
+0.57 \\
+0.58 \\
+0.59\end{array}$ & $\begin{array}{l}+12.3 \\
+9.4 \\
+8.2 \\
+7.5\end{array}$ \\
\hline $\begin{array}{l}21 \\
25 \\
27\end{array}$ & $\begin{array}{rrr}7 & 16 & 54 \\
7 & 2 & 58 \\
6 & 43 & 49\end{array}$ & $\begin{array}{llll}18 & 50 & 52.83 \\
18 & 50 & 34.39 \\
18 & 50 & 28.29\end{array}$ & $\begin{array}{rrr}+0 & 26 & 10.7 \\
-2 & 41 & 1.4 \\
-4 & 7.59 .5\end{array}$ & $\begin{array}{rr}-1 & 16.37 \\
+2 & 8.60 \\
+2 & 11.10\end{array}$ & $\begin{array}{rr}-1 & 11.3 \\
-8 & 7.1 \\
+2 & 3.6\end{array}$ & $\begin{array}{l}\mathrm{t} 25,5 \\
\mathrm{t} 10,2 \\
\mathrm{t} 25,5\end{array}$ & $\begin{array}{l}9.624 \\
9.626 \\
9.618\end{array}$ & $\begin{array}{l}0.739 \\
0.749 \\
0.755\end{array}$ & $\begin{array}{l}+0.61 \\
+0.61 \\
+0.62\end{array}$ & $\begin{array}{l}+6.3 \\
+5.2 \\
+\quad 4.7\end{array}$ \\
\hline $\begin{array}{ll}\text { Dec. } & 2 \\
& 3\end{array}$ & $\begin{array}{lll}6 & 12 & 36 \\
6 & 17 & 33\end{array}$ & $\begin{array}{lll}18 & 50 & 18.40 \\
18 & 50 & 17.26\end{array}$ & $\begin{array}{rrr}-7 & 30 & 35.2 \\
-8 & 9 & 5.0\end{array}$ & $\begin{array}{lr}+1 & 3.25 \\
-0 & 49.51\end{array}$ & $\begin{array}{r}415.7 \\
+\quad 253.9\end{array}$ & $\begin{array}{l}\mathrm{t} 25,5 \\
\mathrm{t} 15,3\end{array}$ & $\begin{array}{l}9.609 \\
9.619\end{array}$ & $\begin{array}{l}0.769 \\
0.768\end{array}$ & $\begin{array}{l}+0.64 \\
+0.66\end{array}$ & $\begin{array}{l}+3.5 \\
+\quad 2.8\end{array}$ \\
\hline \multicolumn{11}{|c|}{$\begin{array}{l}\text { Note.-Oct. 20. Sky unusually clear. A rift seemed to be on each side of tail. Two condensations in head. Tail } 1 \frac{1}{2}{ }^{\circ} \text { long. } \\
\text { BORRELLY (1909 a) }\end{array}$} \\
\hline \begin{tabular}{cc|}
\multicolumn{1}{c|}{1909} \\
June & 18 \\
& 19 \\
& 20 \\
& 25 \\
& 29 \\
July & 19
\end{tabular} & $\begin{array}{rrr}15 & 22 & 47 \\
15 & 14 & 18 \\
15 & 3 & 49 \\
15 & 6 & 38 \\
15 & 12 & 6 \\
14 & 37 & 57\end{array}$ & $\begin{array}{llll}1 & 48 & 28.12 \\
1 & 51 & 23.47 \\
1 & 54 & 22.97 \\
2 & 10 & 31.72 \\
2 & 24 & 46 . & 15 \\
3 & 52 & 24.48\end{array}$ & $\begin{array}{rrr}+33 & 15 & 59.6 \\
+34 & 37 & 36.2 \\
+35 & 57 & 14.5 \\
+42 & 10 & 3.1 \\
+46 & 34 & 10.4 \\
+61 & 47 & 25.0\end{array}$ & $\begin{array}{rr}-1 & 9.59 \\
-2 & 22.97 \\
+1 & 14.67 \\
-1 & 28.61 \\
-0 & 31.06 \\
-0 & 21.28\end{array}$ & $\begin{array}{rrr}- & 1 & 48.0 \\
- & 2 & 49.7 \\
+ & 8 & 51.8 \\
+ & 0 & 2.8 \\
- & 3 & 56.2 \\
- & 0 & 52.7\end{array}$ & $\begin{array}{l}\mathrm{t} 20,4 \\
\mathrm{t} 20,4 \\
\mathrm{t} 30,6 \\
\mathrm{t} 29,6 \\
\mathrm{t} 35,7 \\
\mathrm{t} 30,6\end{array}$ & $\begin{array}{l}9.709 n \\
9.721 n \\
9.734 n \\
9.769 n \\
9.797 n \\
9.980 n\end{array}$ & $\begin{array}{l}0.516 \\
0.516 \\
0.522 \\
0.426 \\
0.324 \\
0.223\end{array}$ & $\begin{array}{l}-0.41 \\
-0.41 \\
-0.38 \\
-0.42 \\
-0.42 \\
-0.44\end{array}$ & $\begin{array}{l}-6.9 \\
-7.0 \\
-7.2 \\
-7.9 \\
-8.4 \\
-8.6\end{array}$ \\
\hline \multicolumn{11}{|c|}{ PERRINE (1909 b) } \\
\hline \begin{tabular}{ll|}
1909 & \\
Oct. & 7 \\
& 8
\end{tabular} & $\begin{array}{rrr}12 & 4 & 55 \\
12 & 25 & 15\end{array}$ & \begin{tabular}{rrr|}
4 & 5 & 56.01 \\
4 & 12 & 30.78
\end{tabular} & $\begin{array}{lrr}+54 & 335.5 \\
+53 & 50 & 41.4\end{array}$ & $\begin{array}{rr}-1 & 3.29 \\
+1 & 41.93\end{array}$ & $\begin{array}{l}-448.1 \\
+451.4\end{array}$ & $\begin{array}{l}\mathrm{t} 12,4 \\
\mathrm{t} 20,6\end{array}$ & $\begin{array}{l}\text { 9. } 734 n \\
9.694 n\end{array}$ & $\begin{array}{l}9.882 n \\
0.008 n\end{array}$ & $\begin{array}{l}+3.38 \\
+3.39\end{array}$ & $\begin{array}{l}+0.9 \\
+0.9\end{array}$ \\
\hline \multicolumn{11}{|c|}{ HALLEY (1909 c) } \\
\hline 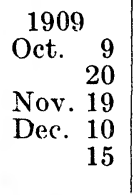 & $\begin{array}{rrr}15 & 18 & 33 \\
13 & 40 & 42 \\
12 & 9 & 31 \\
8 & 35 & 46 \\
8 & 52 & 17\end{array}$ & $\begin{array}{rrr}6 & 14 & 41.15 \\
6 & 6 & 36.69 \\
5 & 8 & 23.65 \\
3 & 47 & 19.16 \\
3 & 25 & 5.65\end{array}$ & 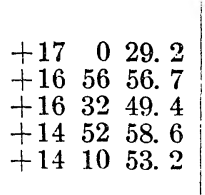 & $\begin{array}{ll}+1 & 50.05 \\
+2 & 18.82 \\
-0 & 44.56 \\
-1 & 25.47 \\
+0 & 59.80\end{array}$ & $\begin{array}{rrr}-9 & 10.1 \\
- & 2 & 27.2 \\
- & 1 & 41.8 \\
- & 6.9 \\
+ & 7 & 34.0\end{array}$ & $\begin{array}{r}\mathrm{t} 5,3 \\
\mathrm{t} 25,5 \\
\mathrm{t} 25,5 \\
\mathrm{t} 25,5 \\
\mathrm{t} 25,5\end{array}$ & $\begin{array}{l}\text { 9. } 317 n \\
9.463 n \\
9.122 n \\
9.357 n \\
9.061 n\end{array}$ & $\begin{array}{l}0.538 \\
0.565 \\
0.531 \\
0.576 \\
0.568\end{array}$ & $\begin{array}{l}+1.87 \\
+2.24 \\
+3.21 \\
+3.35 \\
+3.28\end{array}$ & $\begin{array}{l}+5.2 \\
+\quad 5.3 \\
+\quad 8.1 \\
+12.9 \\
+14.1\end{array}$ \\
\hline
\end{tabular}


DANIEL $(1907 \mathrm{~d})$

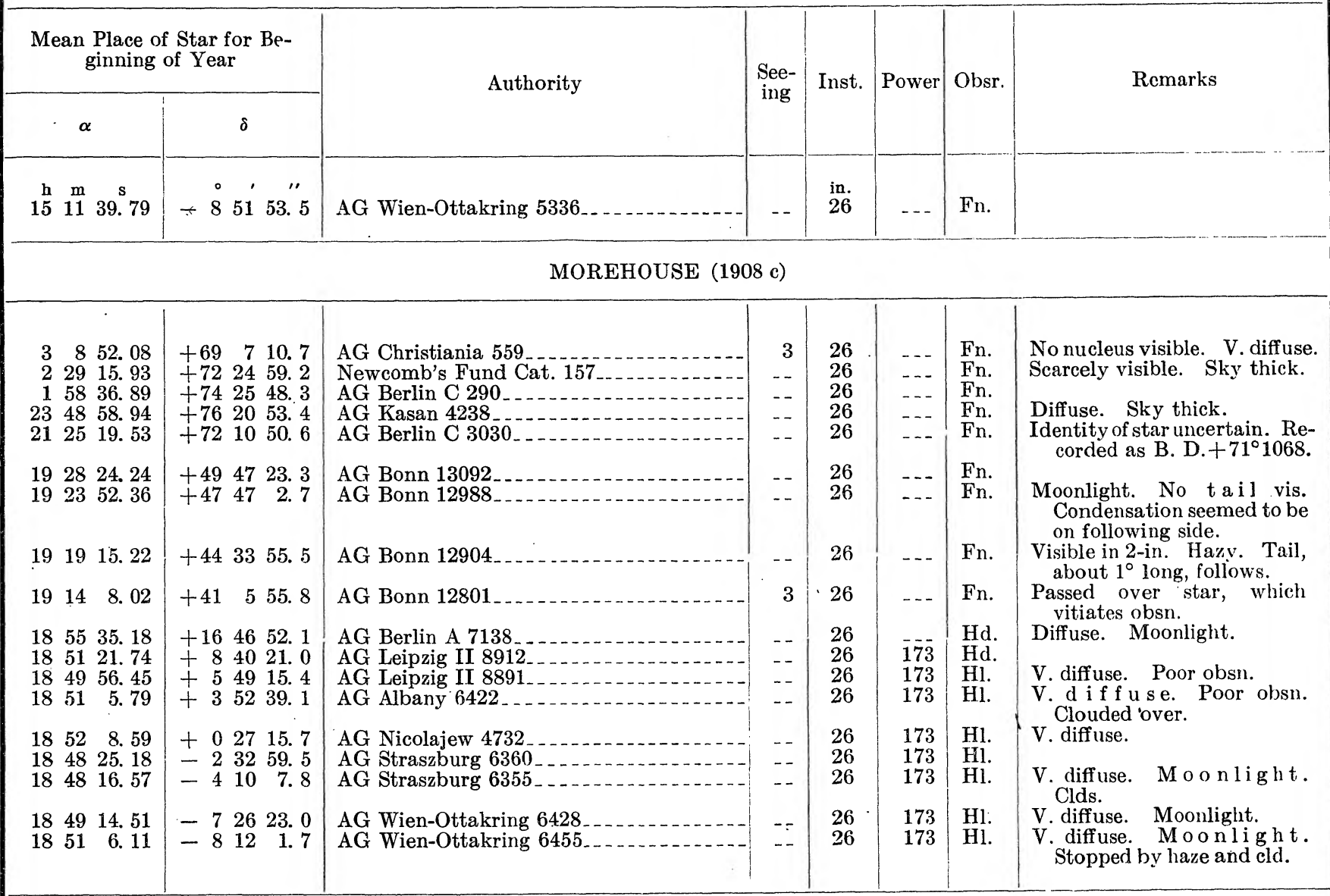

BORRELLY (1909 a)

\begin{tabular}{|c|c|c|c|c|c|c|c|}
\hline $\begin{array}{llr}1 & 49 & 38.12 \\
1 & 53 & 46.85 \\
1 & 53 & 8.68 \\
2 & 12 & 0.75 \\
2 & 25 & 17.63 \\
3 & 52 & 46.20\end{array}$ & 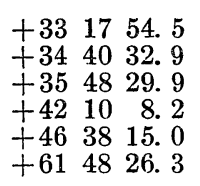 & $\begin{array}{l}\text { AG Leiden 707 } \\
\text { AG Leiden 744 } \\
\text { AG Lund 905 } \\
\text { AG Bonn 1952 } \\
\text { AG Bonn 2118 } \\
\text { AG Hels 3297 }\end{array}$ & $\begin{array}{l}-- \\
-- \\
-- \\
-- \\
--\end{array}$ & $\begin{array}{l}26 \\
26 \\
26 \\
26 \\
26 \\
26\end{array}$ & $\begin{array}{l}-- \\
-- \\
--- \\
--- \\
--- \\
--\end{array}$ & $\begin{array}{l}\text { Fn. } \\
\text { Fn. } \\
\text { Fn. } \\
\text { Fn. } \\
\text { Fn. } \\
\text { Fn. }\end{array}$ & $\begin{array}{l}\text { Ft. Dawn. } \\
\text { Ft. Dawn. } \\
\text { V. v. ft. }\end{array}$ \\
\hline
\end{tabular}

PERRINE (1909 b)

\begin{tabular}{|c|c|c|c|c|c|c|c|}
\hline $\begin{array}{rrr}4 & 6 & 55.92 \\
4 & 10 & 45.46\end{array}$ & $\begin{array}{r}+54822.7 \\
+534549.1\end{array}$ & $\begin{array}{l}\text { AG Harvard 1757 } \\
\text { AG Harvard } 1785\end{array}$ & -- & $\begin{array}{l}26 \\
26\end{array}$ & -- & $\begin{array}{l}\text { Fn. } \\
\text { Fn. }\end{array}$ & V. v. ft. \\
\hline \multicolumn{8}{|c|}{ HALLEY (1909 c) } \\
\hline $\begin{array}{rrr}6 & 12 & 49.23 \\
6 & 4 & 15.63 \\
5 & 9 & 5.00 \\
3 & 48 & 41.28 \\
3 & 24 & 2.57\end{array}$ & $\begin{array}{rrr}+17 & 9 & 34.1 \\
+16 & 59 & 18.6 \\
+16 & 34 & 23.1 \\
+14 & 54 & 52.6 \\
+14 & 3 & 5.1\end{array}$ & $\begin{array}{l}\text { AG Berlin A 2051 } \\
\text { AG Berlin A 1941- } \\
\text { AG Berlin A 1433:- } \\
\text { AG Leipzig I 1132- } \\
\text { AG Leipzig I 1018 }\end{array}$ & $\begin{array}{l}-- \\
-- \\
-- \\
--\end{array}$ & $\begin{array}{l}26 \\
26 \\
26 \\
26 \\
26\end{array}$ & $\begin{array}{l}178 \\
-- \\
\cdots \\
\cdots \\
--\end{array}$ & $\begin{array}{l}\text { Fn. } \\
\text { Ep. } \\
\text { Ep. } \\
\text { Ep. } \\
\text { Ep. }\end{array}$ & V. v. ft. \\
\hline
\end{tabular}


EQUATORIAL OBSERVATIONS，1908-1926

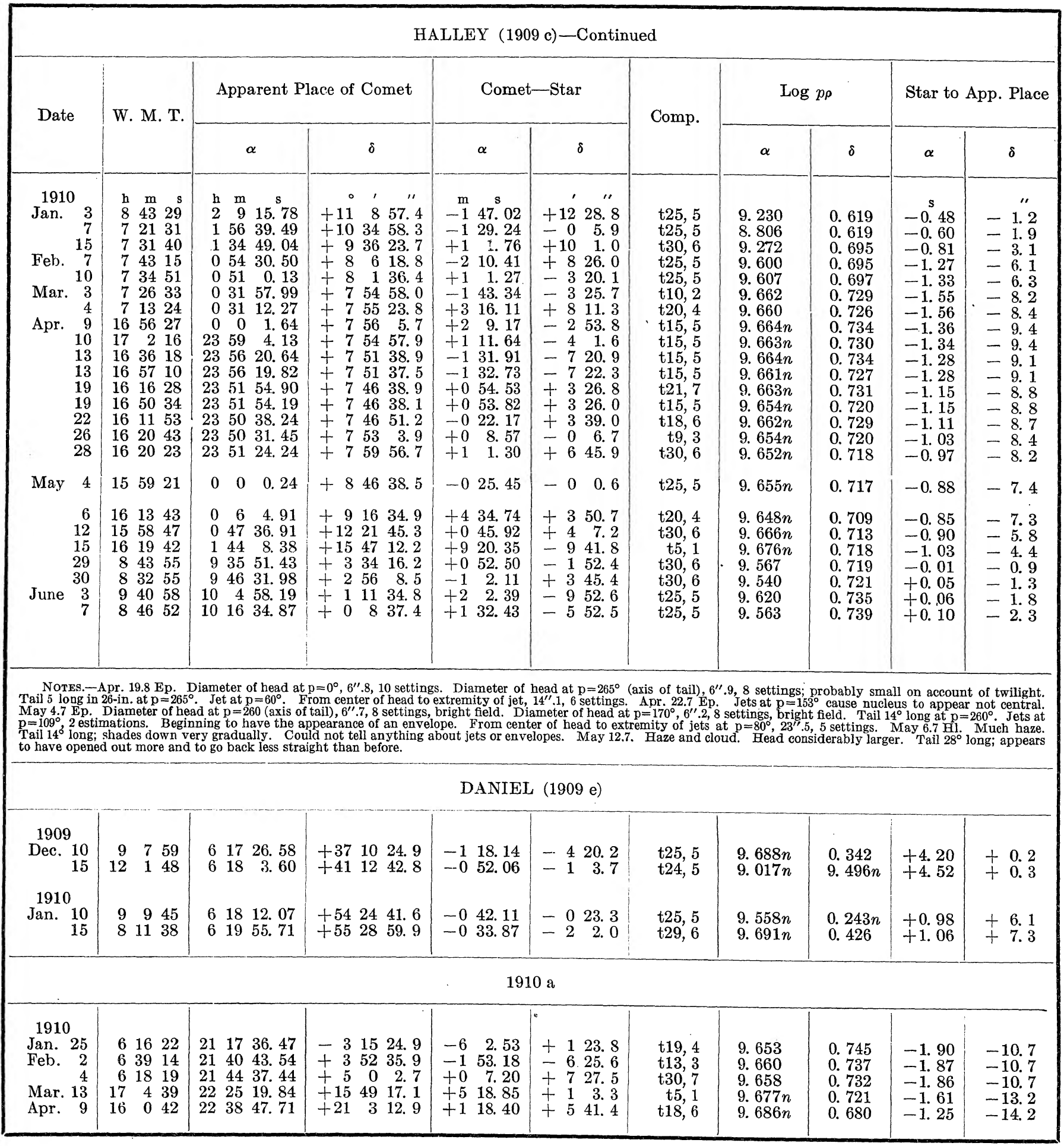


HALLEY (1909 c)-Continued

Mean Place of Star for Beginning of Year

\begin{tabular}{|c|c|}
\hline$\alpha$ & $\delta$ \\
\hline \begin{tabular}{rrr}
$\mathrm{h}$ & $\mathrm{m}$ & \multicolumn{1}{c}{$\mathrm{s}$} \\
2 & 11 & 3.28 \\
1 & 58 & 9.33 \\
1 & 33 & 48.09 \\
0 & 56 & 42.18 \\
0 & 50 & 0.19 \\
0 & 33 & 42.88 \\
0 & 27 & 57.72 \\
23 & 57 & 53.83 \\
23 & 57 & 53.83 \\
23 & 57 & 53.83 \\
23 & 57 & 53.83 \\
23 & 51 & 1.52 \\
23 & 51 & 1.52 \\
23 & 51 & 1.52 \\
23 & 50 & 23.91 \\
23 & 50 & 23.91
\end{tabular} & \begin{tabular}{rrr} 
& $\circ$ & \multicolumn{1}{r}{} \\
+10 & 56 & 29.8 \\
+10 & 35 & 6.1 \\
+ & 96 & 25.8 \\
+7 & 57 & 58.9 \\
+ & 5 & 2.8 \\
+7 & 58 & 31.9 \\
+7 & 47 & 20.9 \\
+7 & 59 & 8.9 \\
+7 & 59 & 8.9 \\
+7 & 59 & 8.9 \\
+7 & 59 & 8.9 \\
+7 & 43 & 20.9 \\
+7 & 43 & 20.9 \\
+7 & 43 & 20.9 \\
+7 & 53 & 19.0 \\
+7 & 53 & 19.0
\end{tabular} \\
\hline $\begin{array}{llll}0 & 0 & 26.57\end{array}$ & +84646.5 \\
\hline $\begin{array}{rrr}0 & 1 & 31.02 \\
0 & 46 & 51.89 \\
1 & 34 & 49.06 \\
9 & 34 & 58.94 \\
9 & 47 & 34.04 \\
10 & 2 & 55.74 \\
10 & 15 & 2.34\end{array}$ & 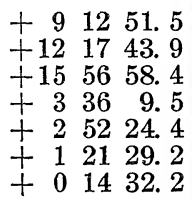 \\
\hline
\end{tabular}

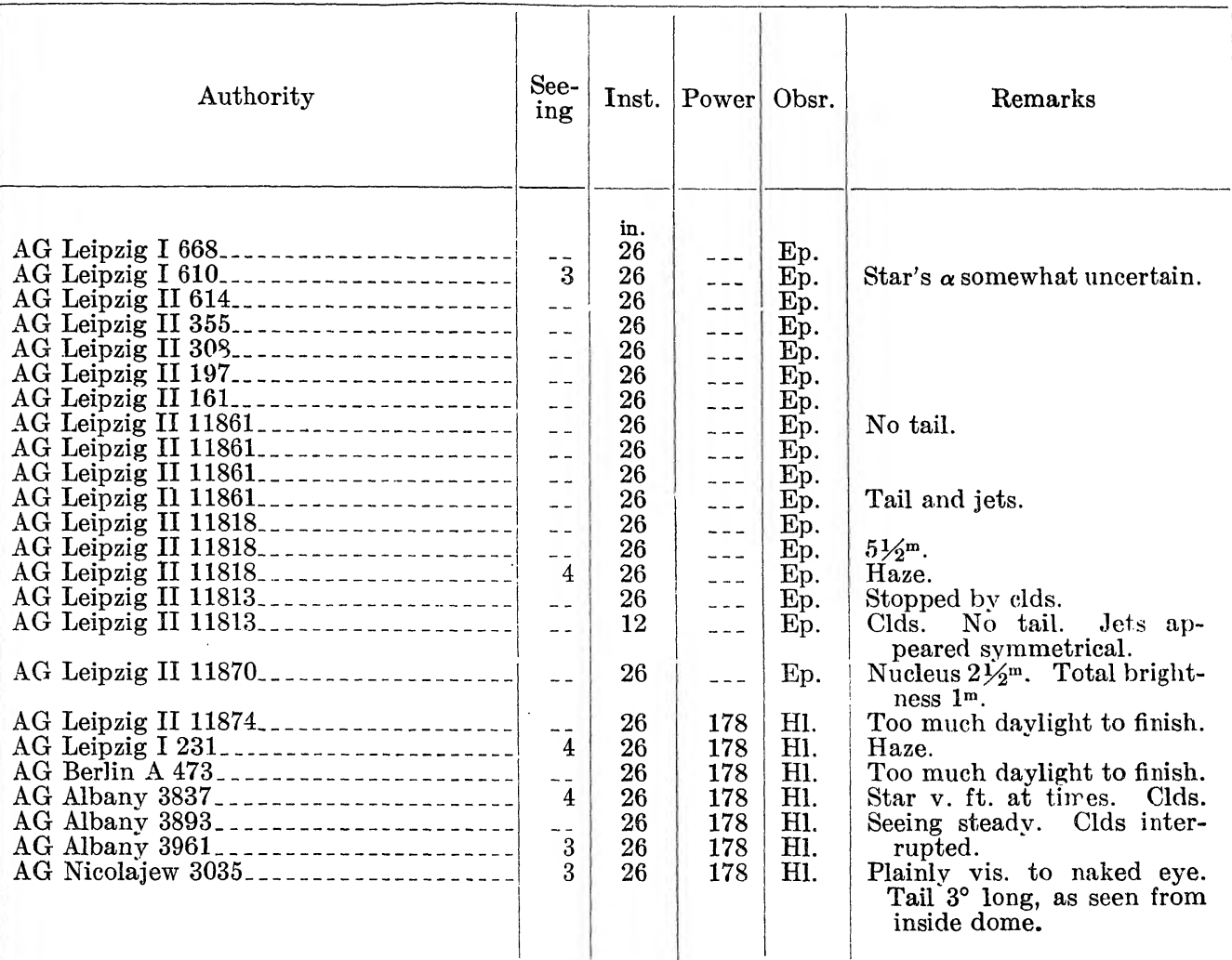

May 15.7. Tail $60^{\circ}$ long; against dark sky before rising of head. May 16.7. Tail $60^{\circ}$ long before rising of head. May 18.6-18.7. Looked for meteor showers, Hall with 26-in., and 5-in. and 2-in. finders, and through slit of large dome; Morgan from ground; Burton and Naulte from top of main building, where city lights interfered. Watch of con halfway between $\nu$ Leonis and $\epsilon$ Leonis; the lower edge passes as far below $\varphi$ Leonis as upper edge above $\epsilon$ Leonis. Tail $45^{\circ}$ long.

DANIEL $(1909$ e)

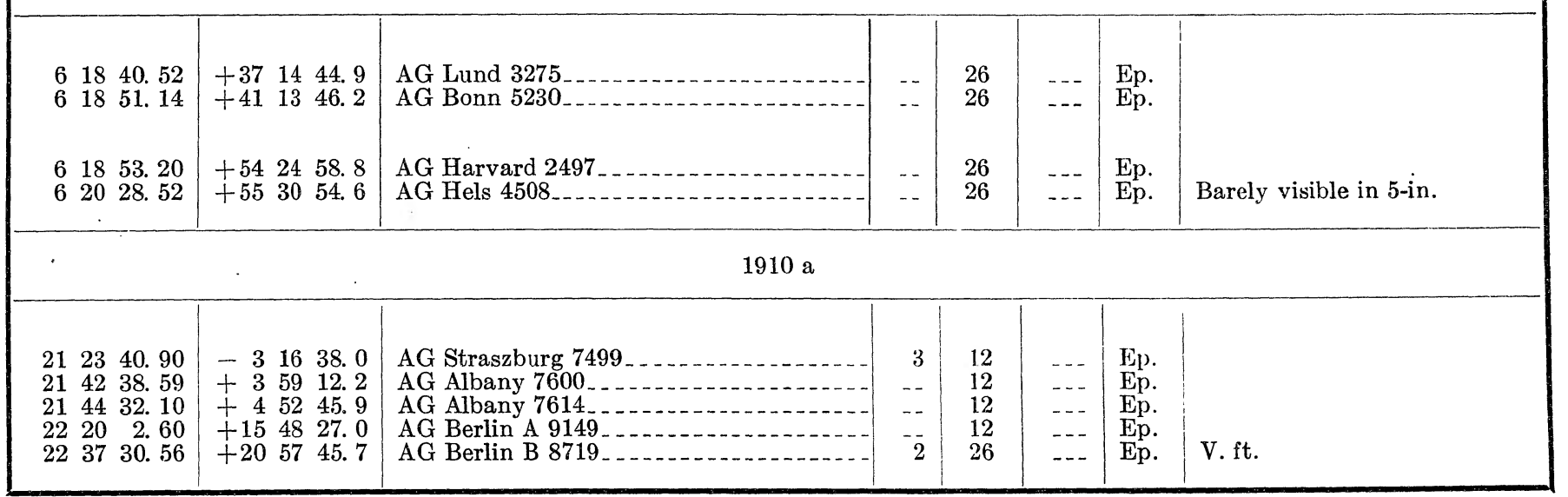




\begin{tabular}{|c|c|c|c|c|c|c|c|c|c|c|}
\hline \multicolumn{11}{|c|}{ METCALF (1910 b) } \\
\hline \multirow{2}{*}{ Date } & \multirow{2}{*}{ W. M. T. } & \multicolumn{2}{|c|}{ Apparent Place of Comet } & \multicolumn{2}{|c|}{ Comet-Star } & \multirow{2}{*}{ Comp. } & \multicolumn{2}{|c|}{$\log p \rho$} & \multicolumn{2}{|c|}{ Star to App. Place } \\
\hline & & $\alpha$ & $\delta$ & $\alpha$ & $\delta$ & & $\alpha$ & $\delta$ & $\alpha$ & $\delta$ \\
\hline $\begin{aligned} & 1910 \\
& \text { Aug. } 10 \\
& 18 \\
& 23 \\
& 30 \\
& \text { Sept. } 26 \\
& 28\end{aligned}$ & $\begin{array}{rrr}\mathrm{h} & \mathrm{m} & \mathrm{s} \\
12 & 4 & 47 \\
9 & 29 & 4 \\
8 & 29 & 11 \\
8 & 1 & 2 \\
8 & 3 & 29 \\
6 & 59 & 22\end{array}$ & $\begin{array}{rrr}\mathrm{h} & \mathrm{m} & \mathrm{s} \\
16 & 10 & 29.33 \\
15 & 55 & 3.67 \\
15 & 47 & 36.45 \\
15 & 39 & 31.43 \\
15 & 26 & 13.99 \\
15 & 26 & 1.81\end{array}$ & 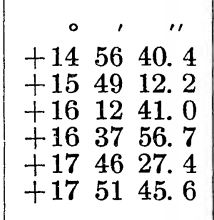 & \begin{tabular}{rr}
$\mathrm{m}$ & \multicolumn{1}{c}{$\mathrm{s}$} \\
-1 & 0.71 \\
-1 & 12.90 \\
-1 & 9.89 \\
-0 & 51.98 \\
-0 & 28.75 \\
-0 & 42.84
\end{tabular} & 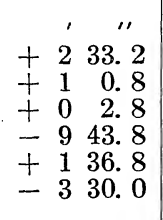 & $\begin{array}{l}\text { t30, } 6 \\
\text { t25, } 5 \\
\text { t25, } 5 \\
\mathrm{t24}, 5 \\
\mathrm{t25}, 5 \\
\mathrm{t25}, 5\end{array}$ & $\begin{array}{l}9.664 \\
9.562 \\
9.502 \\
9.518 \\
9.665 \\
9.620\end{array}$ & $\begin{array}{l}0.692 \\
0.612 \\
0.586 \\
0.585 \\
0.669 \\
0.622\end{array}$ & $\begin{array}{r}\text { s } \\
+1.43 \\
+1.25 \\
+1.12 \\
+0.97 \\
+0.49 \\
+0.47\end{array}$ & $\begin{array}{l} \\
+11 \\
+\quad 2.4 \\
+\quad 2.9 \\
+2.9 \\
+\quad 3.1 \\
+\quad 1.3 \\
+\quad 1.0\end{array}$ \\
\hline \multicolumn{11}{|c|}{ D'ARREST (1910 c) } \\
\hline $\begin{array}{l}1910 \\
\text { Aug. } 30\end{array}$ & $\begin{array}{lll}9 & 26 & 25\end{array}$ & $\begin{array}{lll}16 & 59 & 20.78\end{array}$ & -115528.4 & $-4 \quad 4.90$ & -026.5 & $\mathrm{t} 25,5$ & 9. 520 & 0.805 & +1.60 & -4.2 \\
\hline \multicolumn{11}{|c|}{ FAYE $(1910 \mathrm{e})$} \\
\hline 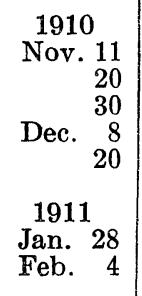 & $\begin{array}{rrr}8 & 47 & 5 \\
7 & 22 & 41 \\
8 & 20 & 47 \\
10 & 53 & 53 \\
9 & 47 & 35\end{array}$ & $\begin{array}{rrr}3 & 38 & 26.95 \\
3 & 37 & 31.22 \\
3 & 36 & 42.34 \\
3 & 37 & 5.46 \\
3 & 40 & 24.03\end{array}$ & $\begin{array}{rrrr}+ & 8 & 9 & 16.7 \\
+ & 6 & 7 & 16.9 \\
+ & 4 & 24 & 14.4 \\
+ & 3 & 32 & 47.6 \\
+ & 3 & 7 & 56.3 \\
& & & \\
+ & 6 & 18 & 2.9 \\
+ & 7 & 10 & 26.5\end{array}$ & $\begin{array}{rr}-1 & 44.28 \\
+2 & 33.78 \\
+0 & 5.66 \\
+2 & 14.25 \\
+1 & 9.56 \\
& \\
+0 & 29.07 \\
-1 & 0.63\end{array}$ & 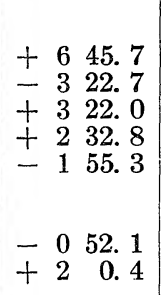 & $\begin{array}{l}\mathrm{t} 30,6 \\
\mathrm{t} 25,5 \\
\mathrm{t} 30,8 \\
\mathrm{t} 25,5 \\
\mathrm{t} 25,5\end{array}$ & $\begin{array}{l}\text { 9. } 564 n \\
9.618 n \\
9.469 n \\
\text { 8. } 696 \\
\text { 7. } 681 \\
\\
\text { 8. } 412 n \\
7.804\end{array}$ & $\begin{array}{l}0.686 \\
0.712 \\
0.706 \\
0.705 \\
0.709 \\
\\
0.673 \\
0.663\end{array}$ & $\begin{array}{l}+3.17 \\
+3.26 \\
+3.31 \\
+3.36 \\
+3.40 \\
\\
+0.18 \\
+0.13\end{array}$ & $\begin{array}{l}+16.8 \\
+16.5 \\
+15.6 \\
+15.1 \\
+14.0 \\
+1.4 \\
+1.6\end{array}$ \\
\hline \multicolumn{11}{|c|}{ KIESS (1911 b) } \\
\hline \begin{tabular}{lr}
\multicolumn{1}{c|}{1911} \\
July & 9 \\
& 12 \\
& 18 \\
Aug. & 9
\end{tabular} & $\begin{array}{rrr}14 & 47 & 44 \\
15 & 3 & 2 \\
15 & 21 & 33 \\
16 & 0 & 52\end{array}$ & $\begin{array}{rrr}4 & 46 & 51.13 \\
4 & 41 & 35.20 \\
4 & 30 & 49.04 \\
3 & 8 & 43.63\end{array}$ & 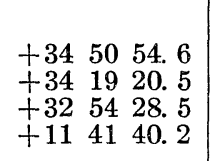 & $\begin{array}{ll}+3 & 27.71 \\
-1 & 15.11 \\
-3 & 32.32 \\
+3 & 20.98\end{array}$ & 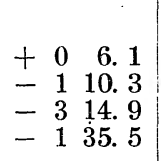 & $\begin{array}{l}\mathrm{t} 25,5 \\
\mathrm{t} 25,5 \\
\mathrm{t} 25,5 \\
\mathrm{t} 25,5\end{array}$ & $\begin{array}{l}9.735 n \\
9.742 n \\
9.731 n \\
9.358 n\end{array}$ & $\begin{array}{l}0.730 \\
0.685 \\
0.608 \\
0.620\end{array}$ & $\begin{array}{l}-0.14 \\
-0.03 \\
+0.16 \\
+1.32\end{array}$ & $\begin{array}{l}+4.0 \\
+4.2 \\
+4.5 \\
+13.4\end{array}$ \\
\hline \multicolumn{11}{|c|}{ BROOKS (1911 c) } \\
\hline \begin{tabular}{lr|}
\multicolumn{2}{c|}{1911} \\
July & 24 \\
& 31 \\
Aug. & 9 \\
Sept. & 1 \\
& 13 \\
& 18 \\
& 26 \\
Oct. & 23
\end{tabular} & $\begin{array}{rrr}13 & 49 & 31 \\
13 & 46 & 24 \\
10 & 6 & 28 \\
10 & 53 & 0 \\
11 & 30 & 2 \\
10 & 49 & 1 \\
8 & 26 & 6 \\
17 & 46 & 40\end{array}$ & $\begin{array}{lrr}22 & 10 & 22.08 \\
22 & 1 & 46.63 \\
21 & 43 & 57.85 \\
19 & 36 & 59.64 \\
17 & 10 & 57.07 \\
16 & 7 & 36.81 \\
14 & 45 & 18.81 \\
12 & 35 & 44.21\end{array}$ & 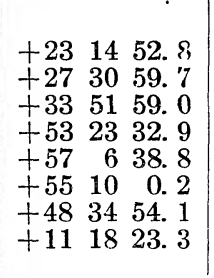 & $\begin{array}{rr}+2 & 2.90 \\
-0 & 6.06 \\
+1 & 16.10 \\
-0 & 9.53 \\
+0 & 6.31 \\
-8 & 7.74 \\
-0 & 52.28 \\
-1 & 57.54\end{array}$ & 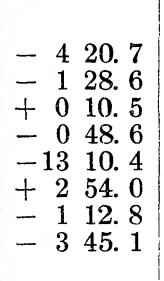 & $\begin{array}{r}\mathrm{t} 25,5 \\
\mathrm{~d} 9,8 \\
\mathrm{t} 25,5 \\
\mathrm{t} 29,6 \\
\mathrm{t} 12,4 \\
\mathrm{t} 15,3 \\
\mathrm{t} 25,5 \\
\mathrm{t} 25,5\end{array}$ & $\begin{array}{l}8.462 n \\
8.646 \\
9.519 n \\
9.573 \\
9.924 \\
9.900 \\
9.840 \\
9.643 n\end{array}$ & $\begin{array}{l}0.371 \\
0.236 \\
0.179 \\
0.183 n \\
0.426 \\
0.586 \\
0.560 \\
0.694\end{array}$ & $\begin{array}{l}+2.13 \\
+2.24 \\
+2.39 \\
+1.98 \\
+0.65 \\
+0.30 \\
+0.12 \\
+0.75\end{array}$ & $\begin{array}{l}+3.1 \\
+4.0 \\
+5.7 \\
+11.7 \\
+10.0 \\
+6.9 \\
+0.4 \\
+8.0\end{array}$ \\
\hline \multicolumn{11}{|c|}{ QUENNISSET (1911 f) } \\
\hline \begin{tabular}{c|}
1911 \\
Sept. 25 \\
26 \\
29 \\
Oct. 16
\end{tabular} & \begin{tabular}{rrr|}
9 & 25 & 12 \\
9 & 10 & 25 \\
8 & 44 & 55 \\
7 & 55 & 2
\end{tabular} & $\begin{array}{rrr}14 & 45 & 22.14 \\
14 & 52 & 5.33 \\
15 & 6 & 39.64 \\
15 & 37 & 5.63\end{array}$ & 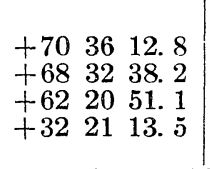 & \begin{tabular}{rr|}
-4 & 49.56 \\
-2 & 11.15 \\
-0 & 8.67 \\
-0 & 58.40
\end{tabular} & $\begin{array}{r}+359.5 \\
+33.1 \\
+213.6 \\
+1250.9\end{array}$ & $\begin{array}{l}\text { t20,4 } \\
\mathrm{t} 25,5 \\
\mathrm{t} 20,4 \\
\mathrm{t} 30,8\end{array}$ & $\begin{array}{l}0.126 \\
0.091 \\
9.993 \\
9.733\end{array}$ & $\begin{array}{l}0.527 \\
0.484 \\
0.446 \\
0.660\end{array}$ & $\begin{array}{l}-1.42 \\
-1.20 \\
-0.66 \\
+0.34\end{array}$ & $\begin{array}{l}+0.5 \\
+0.7 \\
+0.9 \\
-\quad 2.2\end{array}$ \\
\hline
\end{tabular}


METCALF (1910 b)

Mean Place of Star for Beginning of Year

\begin{tabular}{|c|c|}
\hline$\alpha$ & $\delta$ \\
\hline $\begin{array}{rcc}\mathrm{h} & \mathrm{m} & \mathrm{s} \\
16 & 11 & 28.61 \\
15 & 56 & 15.32 \\
15 & 48 & 45.22 \\
15 & 40 & 22.44 \\
15 & 26 & 42.25 \\
15 & 26 & 44.18\end{array}$ & \begin{tabular}{rrr}
$\circ$ & \multicolumn{1}{c}{} \\
+1454 & 4.8 \\
+1548 & 8.5 \\
+16 & 12 & 35.3 \\
+1647 & 37.4 \\
+17 & 44 & 49.3 \\
+17 & 55 & 14.6
\end{tabular} \\
\hline
\end{tabular}

\begin{tabular}{|c|c|c|c|c|c|}
\hline Authority & $\begin{array}{l}\text { See- } \\
\text { ing }\end{array}$ & Inst. & Power & Obsr. & Remarks \\
\hline $\begin{array}{l}\text { AG Leipzig I 5661; AG Berlin A 5818 } \\
\text { AG Berlin A 5725 } \\
\text { AG Berlin A 5675 } \\
\text { AG Berlin A 5632 } \\
\text { AG Berlin A 5564 Berlin A 5565 }\end{array}$ & $\begin{array}{l}-- \\
-\overline{5} \\
-- \\
-- \\
--\end{array}$ & $\begin{array}{l}\text { in. } \\
26 \\
26 \\
12 \\
26 \\
26 \\
26\end{array}$ & $\begin{array}{l}--- \\
-- \\
-- \\
-- \\
-- \\
--\end{array}$ & $\begin{array}{l}\text { Bn. } \\
\text { Ep. } \\
\text { Ep. } \\
\text { Ep. } \\
\text { Bn. } \\
\text { Ep. }\end{array}$ & $\begin{array}{l}\text { V. ft. } \\
\text { V.ft. Thick haze. Poor obsn. } \\
\text { Visible in 5-in. }\end{array}$ \\
\hline
\end{tabular}

D'ARREST (1910 c)

\begin{tabular}{lll|lll|l}
17 & 3 & 24.08 & -11 & 54 & 57.7 & Radcliffe 4456
\end{tabular} \begin{tabular}{l|l|l|ll}
26 & $\ldots$ & Ep. & V. v. ft. & Poor obsn.
\end{tabular}

FAYE (1910 e $)$

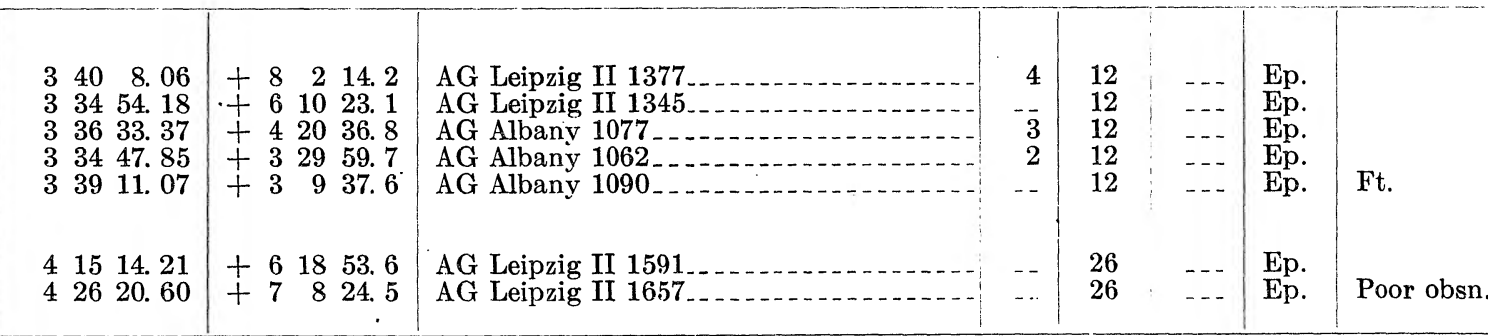

KIESS (1911 b)

$44323.56+345044.5$

$44250.34+342026.6$

$43421.20+325738.9$

$\begin{array}{lllll}3 & 5 & 21.33 & & \end{array}$
AG Leiden 1778

$A G$ Leiden 1775

AG Leiden 1747

AG Leipzig I 948

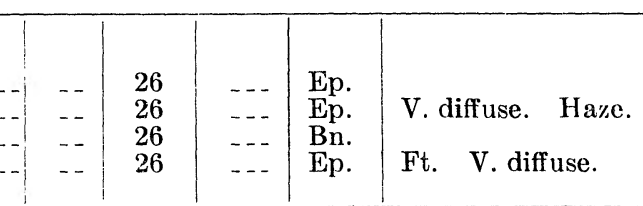

BROOKS (1911 e)

\begin{tabular}{rrrr|rrr|}
\hline 22 & 8 & 17.05 & +23 & 19 & 10.4 \\
22 & 1 & 50.45 & +27 & 32 & 24.3 \\
21 & 42 & 39.36 & +33 & 51 & 42.8 \\
19 & 37 & 7.19 & +53 & 24 & 9.8 \\
17 & 10 & 50.11 & +57 & 19 & 39.2 \\
16 & 15 & 44.25 & +55 & 6 & 59.3 \\
14 & 46 & 10.97 & +48 & 36 & 6.5 \\
12 & 37 & 41.00 & +11 & 22 & 16.4 \\
\hline
\end{tabular}

\begin{tabular}{|l|l|} 
& \\
AG Berlin B 8559 & \\
AG Cambridge 13179 & \\
AG Leiden 9120 & \\
AG Harvard 6130 & \\
AG Hels 9172 & \\
AG Hels 8737 Bonn 9627 & \\
AG Leipzig I 4651
\end{tabular}

\begin{tabular}{l|l|} 
& \\
-- & 26 \\
-- & 26 \\
-- & 26 \\
-- & 12 \\
-- & 26 \\
-- & 26 \\
-- & 26 \\
-- & 26
\end{tabular}

\begin{tabular}{l|l} 
& \\
$\ldots$ & Bn. \\
$\ldots \ldots$ & Ep. \\
$\cdots \cdots$ & Ep. \\
\hdashline- & Ep. \\
\hdashline- & Ep. \\
\hdashline- & Ep. \\
\hdashline- & Ep. \\
$\ldots$ & Bn.
\end{tabular}

Ft. Moonlight.

Easily visible to naked eye. Nucleus well defined.

QUÉNISSET (1911 f)

$145013.12+703212.8$ $\begin{array}{llll}14 & 54 & 17.68\end{array}$

$\begin{array}{lll}15 & 6 & 48.97\end{array}$

$\begin{array}{lrr}15 & 38 & 3.69\end{array}$
$+683545.6$

$+621836.6$

$+32824.8$
AG Berlin C 2011

A G Christiania 2227

Comp, with AG Hels 8270

AG. Leiden 5560

\begin{tabular}{c|r} 
& \\
-- & 26 \\
-- & 26 \\
-- & 26 \\
2 & 12
\end{tabular}

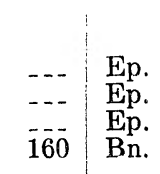




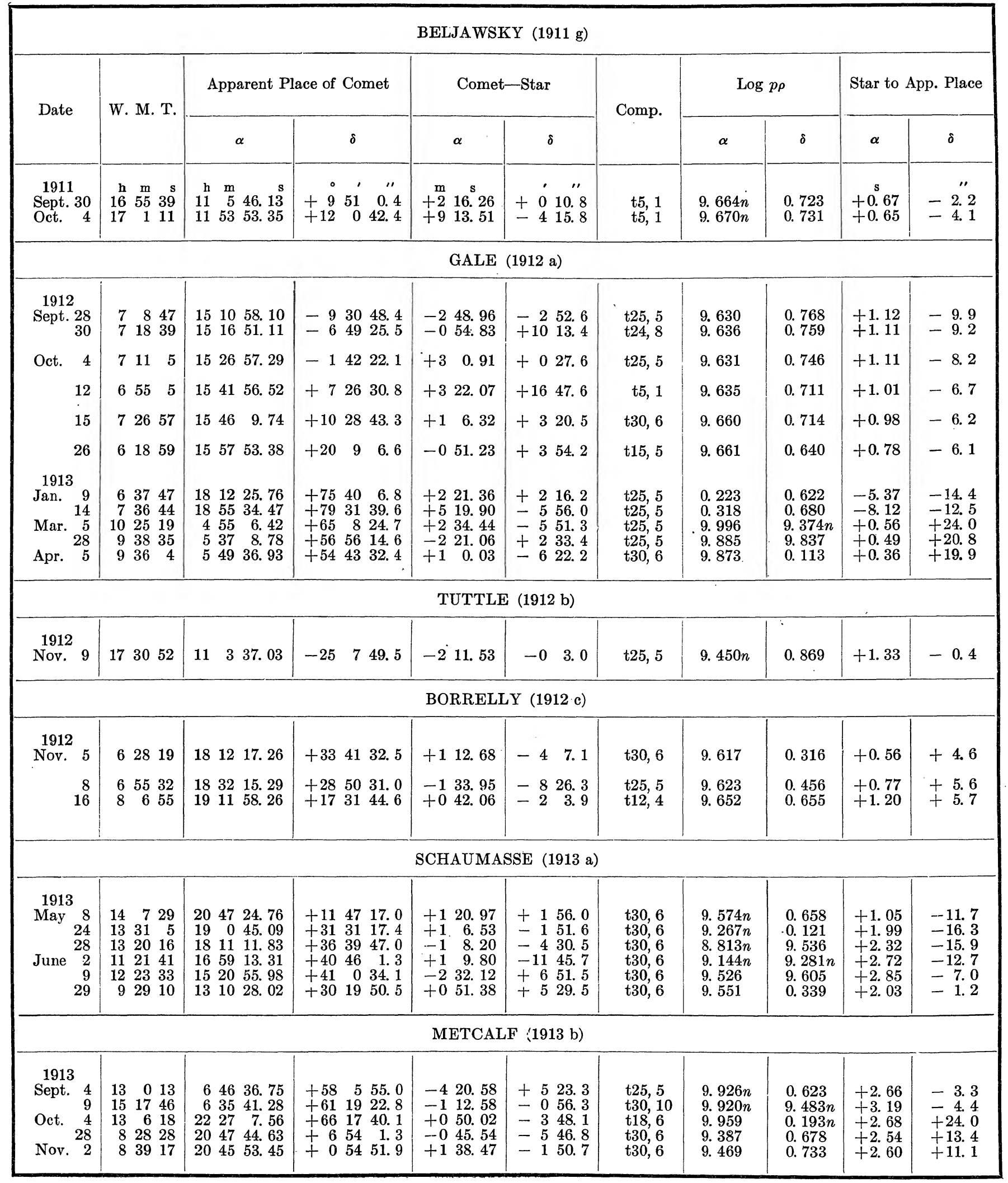




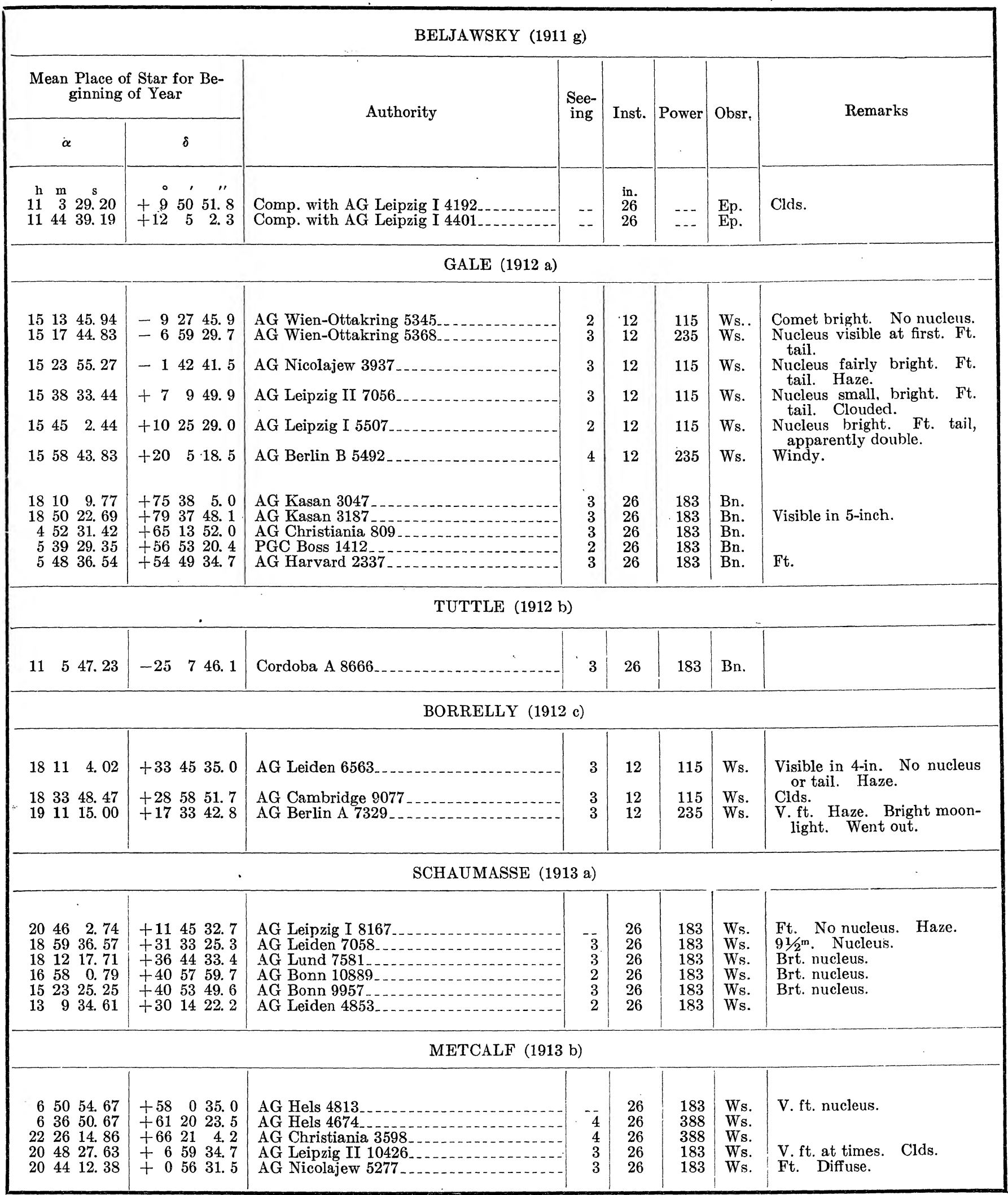




\begin{tabular}{|c|c|c|c|c|c|c|c|c|c|c|}
\hline \multicolumn{11}{|c|}{ NEUJMIN (1913 c) } \\
\hline \multirow{2}{*}{ Date } & \multirow{2}{*}{ W. M. T. } & \multicolumn{2}{|c|}{ Apparent Place of Comet } & \multicolumn{2}{|c|}{ Comet-Star } & \multirow{2}{*}{ Comp. } & \multicolumn{2}{|c|}{$\log p \rho$} & \multicolumn{2}{|c|}{ Star to App. Place } \\
\hline & & $\alpha$ & $\delta$ & $\alpha$ & $\delta$ & & $\alpha$ & $\delta$ & $\alpha$ & $\delta$ \\
\hline $\begin{array}{l}1913 \\
\text { Sept. } 9\end{array}$ & $\begin{array}{rrr}\mathrm{h} & \mathrm{m} & \mathrm{s} \\
13 & 15 & 27\end{array}$ & $\begin{array}{rrc}\mathrm{h} & \mathrm{m} & \mathrm{s} \\
23 & 48 & 1.55\end{array}$ & $\begin{array}{r}\circ{ }^{\prime}{ }^{\prime \prime} \\
+05921.6\end{array}$ & $\begin{array}{cc}\mathrm{m} & \mathrm{s} \\
+0 & 19.33\end{array}$ & $\begin{array}{c}\prime \prime \prime \\
-211.8\end{array}$ & $\mathrm{t} 30,10$ & 8. 922 & 0.731 & $\begin{array}{l}\mathrm{s} \\
+3.48\end{array}$ & $\begin{array}{c}\prime \prime \\
+22.1\end{array}$ \\
\hline 22 & $\begin{array}{lll}11 & 23 & 3\end{array}$ & 233951.90 & +61839.8 & +17.95 & -129.6 & $\mathrm{t} 30,10$ & 8. $349 n$ & 0.673 & +3.51 & +22.5 \\
\hline 23 & $11 \quad 1634$ & $\begin{array}{llll}23 & 39 & 17 . & 10\end{array}$ & +64018.6 & $-1 \quad 8.93$ & -236.4 & $\mathrm{t} 30,10$ & 8. $420 n$ & 0.669 & +3.52 & +22.7 \\
\hline 24 & 112847 & $\begin{array}{lll}23 & 38 & 42.60\end{array}$ & +7145.9 & $-0 \quad 30.36$ & -252.4 & $\mathrm{t} 30,10$ & 7. 865 & 0.664 & +3.52 & +22.8 \\
\hline Oct. 22 & $1029 \quad 0$ & $23 \quad 33 \quad 40.83$ & +14133.0 & +053.47 & +249.4 & $\mathrm{t} 30,10$ & 9. 080 & 0.572 & +3.41 & +25.3 \\
\hline \multicolumn{11}{|c|}{ WESTPHAL (1913 d) } \\
\hline $\begin{array}{l}1913 \\
\text { Sept. } 27 \\
\text { Oct. } 4\end{array}$ & $\begin{array}{rrr}12 & 9 & 6 \\
9 & 48 & 58\end{array}$ & $\begin{array}{llll}21 & 50 & 37.66 \\
21 & 29 & 24.93 \\
\end{array}$ & $\begin{array}{r}13732.7 \\
+\quad 41730.6\end{array}$ & $\begin{array}{l}+258.76 \\
+033.23\end{array}$ & $\begin{array}{l}+346.5 \\
+636.3\end{array}$ & $\begin{array}{l}\text { t30, } 6 \\
\text { t30. } 10\end{array}$ & $\begin{array}{l}9.477 \\
9.153\end{array}$ & $\begin{array}{l}0.752 \\
0.698\end{array}$ & $\begin{array}{l}+3.40 \\
+3.14\end{array}$ & $\begin{array}{l}+15.5 \\
+15: 7\end{array}$ \\
\hline \multicolumn{11}{|c|}{ GIACOBINI (1913 e) } \\
\hline $\begin{array}{lr}1913 & \\
\text { Oct. } & 26 \\
\text { Nov. } & 1 \\
4 \\
6\end{array}$ & $\begin{array}{rrr}7 & 59 & 28 \\
8 & 6 & 50 \\
7 & 27 & 52 \\
7 & 28 & 42\end{array}$ & $\begin{array}{rrr}18 & 56 & 28.12 \\
19 & 27 & 13.67 \\
19 & 44 & 6.48 \\
19 & 56 & 6.24\end{array}$ & $\begin{array}{rrr}7 & 26 & 49.8 \\
-13 & 13 & 55.1 \\
-16 & 13 & 42.1 \\
-18 & 15 & 47.0\end{array}$ & $\begin{array}{rr}+0 & 38.19 \\
+1 & 8.64 \\
-2 & 28.57 \\
+2 & 1.32\end{array}$ & $\begin{array}{rr}+3 & 26.6 \\
-4 & 4.2 \\
-8 & 17.1 \\
-4 & 4.4\end{array}$ & $\begin{array}{l}\text { t24, } 8 \\
\mathrm{t} 36,6 \\
\mathrm{t} 25,5 \\
\mathrm{t} 30,6\end{array}$ & $\begin{array}{l}9.551 \\
9.560 \\
9.482 \\
9.479\end{array}$ & $\begin{array}{c}0.779 \\
0.803 \\
0.830 \\
0.839 \\
.\end{array}$ & $\begin{array}{r}+2.27 \\
+2.48 \\
+2.64 \\
+2.70\end{array}$ & $\begin{array}{l}-0.6 \\
-0.1 \\
+\quad 0.4 \\
+\quad 0.2\end{array}$ \\
\hline \multicolumn{11}{|c|}{ DELAVAN (1913 f) } \\
\hline $\begin{array}{l}1913 \\
\text { Dec. } 18\end{array}$ & $\begin{array}{lll}8 & 35 & 47\end{array}$ & $\begin{array}{lll}3 & 2 & 26.93\end{array}$ & $-7 \quad \begin{array}{llll}-7 & 57.1\end{array}$ & -358.67 & +315.1 & $\mathrm{t} 25,5$ & 8. $886 n$ & 0.800 & +4.13 & +19.1 \\
\hline 19 & $\begin{array}{llll}7 & 10 & 56\end{array}$ & $\begin{array}{lll}3 & 1 & 37.21\end{array}$ & -71430.9 & +127.17 & -247.7 & $\mathrm{t} 25,5$ & 9. $358 n$ & 0.793 & +4.10 & +19.2 \\
\hline 22 & $\begin{array}{lll}7 & 42 & 29\end{array}$ & $259 \quad 1.69$ & -6569.0 & +16.77 & -628.4 & $\mathrm{t} 25,5$ & 9. $157 n$ & 0.795 & +4.07 & +18.9 \\
\hline 26 & $\begin{array}{lll}8 & 59 & 36\end{array}$ & 25545.66 & -62936.9 & +323.70 & +617.6 & $\mathrm{t} 10,2$ & 8. 676 & 0. 795 & +4.04 & +18.6 \\
\hline 27 & $\begin{array}{lll}7 & 59 & 4\end{array}$ & $255 \quad 1.50$ & -6232.8 & -055.77 & +24.9 & $\mathrm{t} 25,5$ & 8. $811 n$ & 0. 794 & +4.06 & +18.5 \\
\hline 29 & $\begin{array}{lll}7 & 50 & 26\end{array}$ & 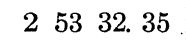 & - 6854.4 & +15.68 & -759.6 & $\mathrm{t} 30,6$ & 8. $801 n$ & 0.792 & +4.04 & +18.6 \\
\hline $\begin{array}{c}1914 \\
\text { Jan. }\end{array}$ & $\begin{array}{lll}8 & 8 & 12\end{array}$ & $\begin{array}{lll}2 & 48 & 50.73\end{array}$ & -51535.4 & +444.78 & +14.1 & $\mathrm{t} 25,5$ & 8. 568 . & 0.785 & +0.94 & +3.2 \\
\hline 11 & $\begin{array}{lll}7 & 32 & 51\end{array}$ & $\begin{array}{lll}2 & 45 & 31.99\end{array}$ & $-426 \quad 3.0$ & +046.94 & +849.5 & $\mathrm{t} 30,6$ & 8. 301 & 0. 779 & +0.90 & +2.9 \\
\hline 18 & 74055 & $\begin{array}{llll}2 & 42 & 29 . & 03\end{array}$ & -32350.1 & -434.14 & -247.8 & $\mathrm{t} 25,5$ & 8.985 & 0.770 & +0.81 & +2.9 \\
\hline 21 & $\begin{array}{lll}7 & 29 & 38\end{array}$ & $241 \quad 27.34$ & $-256 \quad 7.4$ & -147.70 & +021.6 & $\mathrm{t} 30,6$ & 8. 998 & 0.766 & +0.76 & +2.7 \\
\hline Mar. $\begin{array}{r}3 \\
14\end{array}$ & \begin{tabular}{lll|}
8 & 34 & 57 \\
7 & 46 & 20
\end{tabular} & $\begin{array}{lrr}2 & 43 & 17.43 \\
2 & 48 & 9.96\end{array}$ & $\begin{array}{rrr}+4 & 9 & 1.5 \\
+ & 611 & 1.6\end{array}$ & $\begin{array}{l}-325.28 \\
+148.90\end{array}$ & $\begin{array}{l}+346.3 \\
+138.1\end{array}$ & $\begin{array}{l}\mathrm{t} 25,5 \\
\mathrm{t} 30,6\end{array}$ & $\begin{array}{l}9.631 \\
9.624\end{array}$ & $\begin{array}{l}0.725 \\
0.714\end{array}$ & $\begin{array}{l}+0.18 \\
+0.04\end{array}$ & $\begin{array}{l}+3.5 \\
+4.1\end{array}$ \\
\hline
\end{tabular}




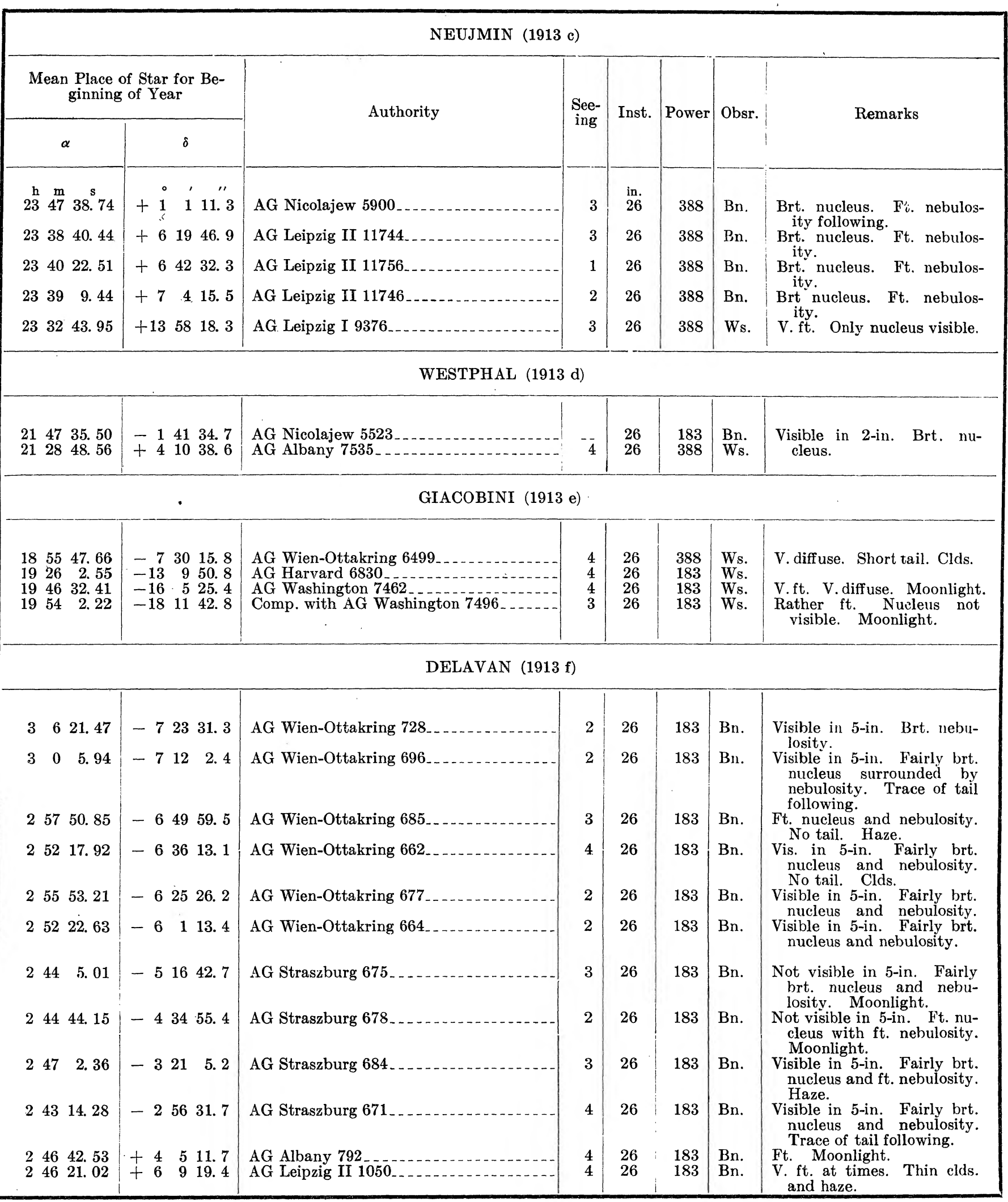




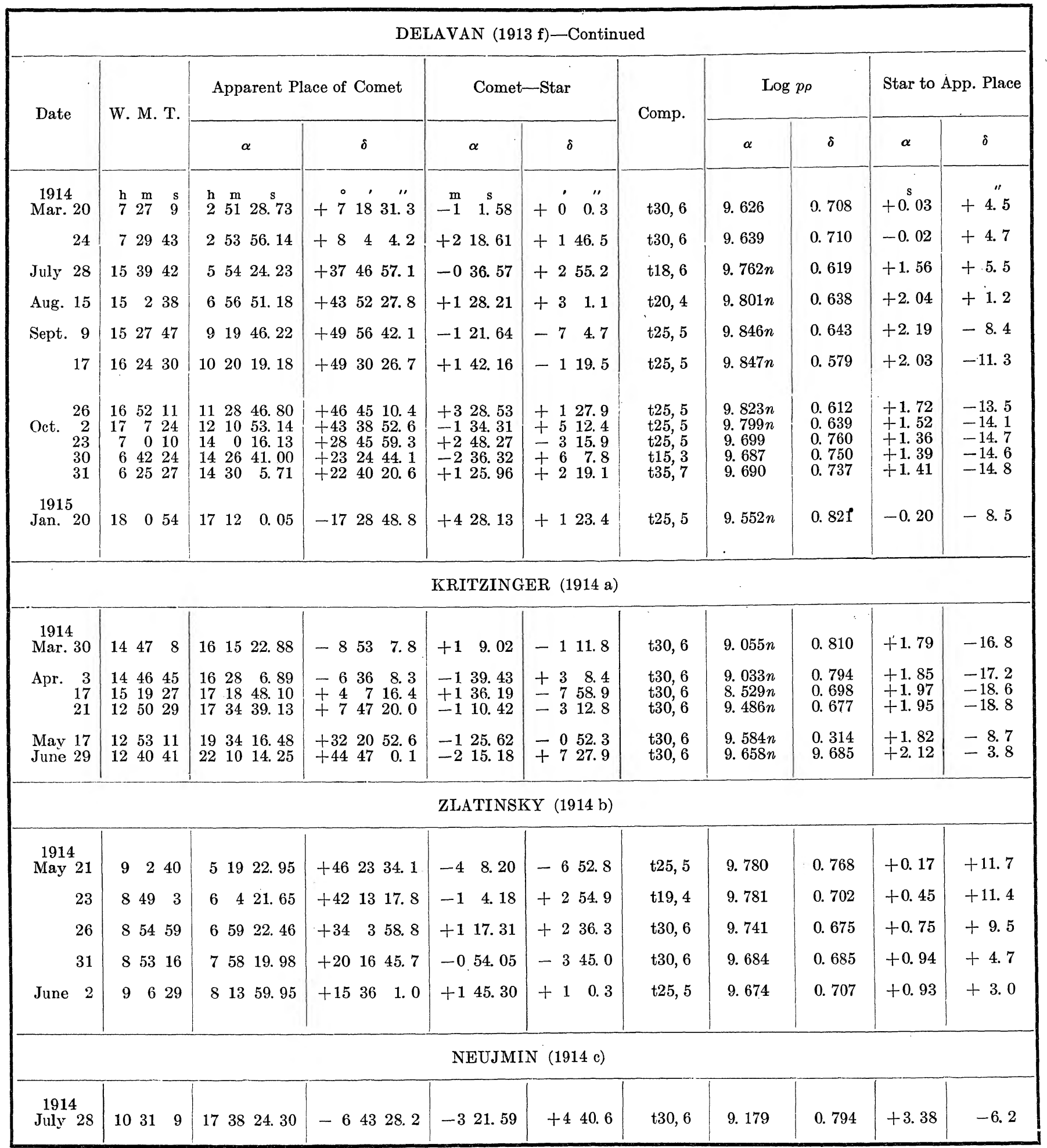


DELAVAN (1913 f)-Continued

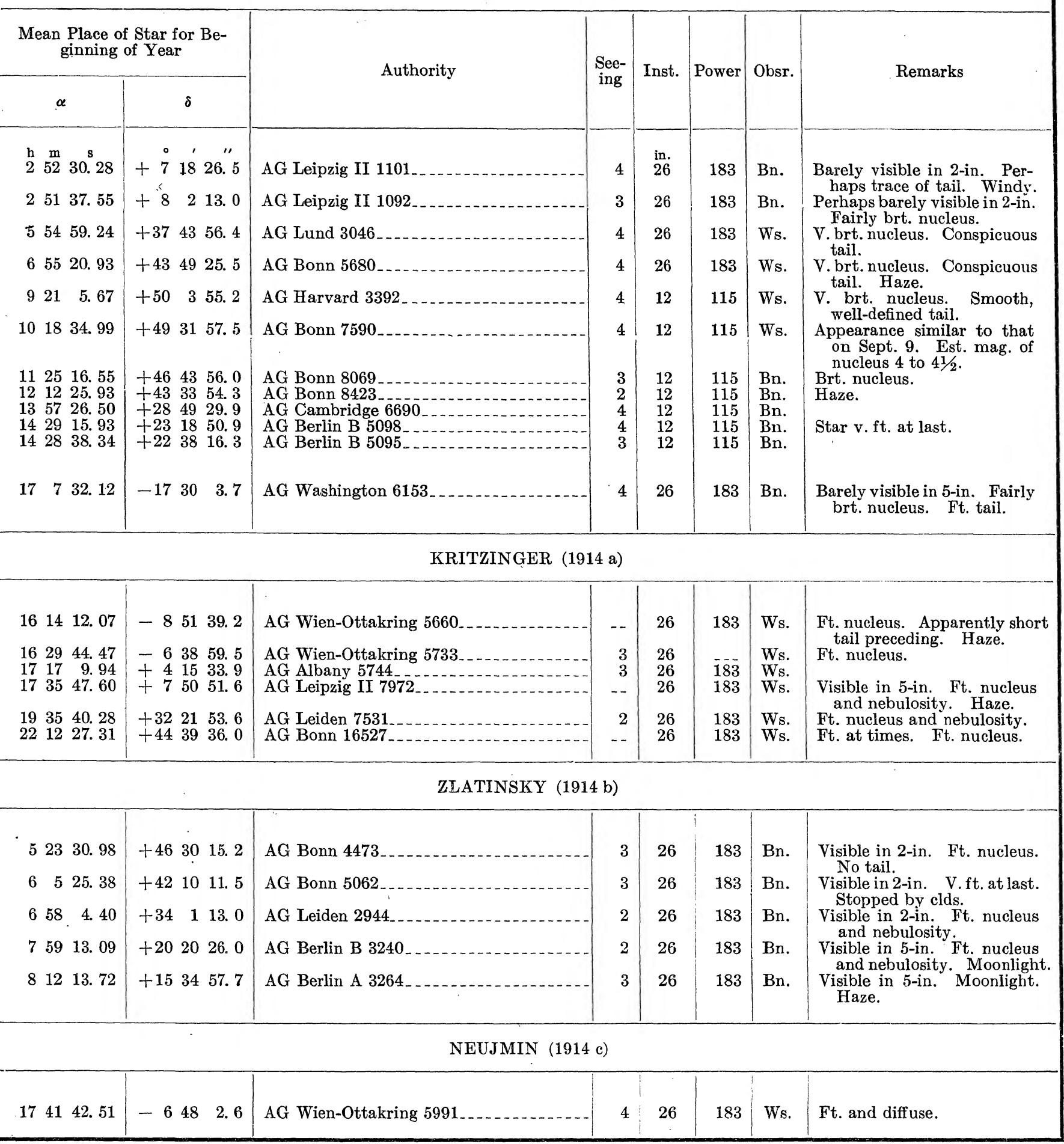




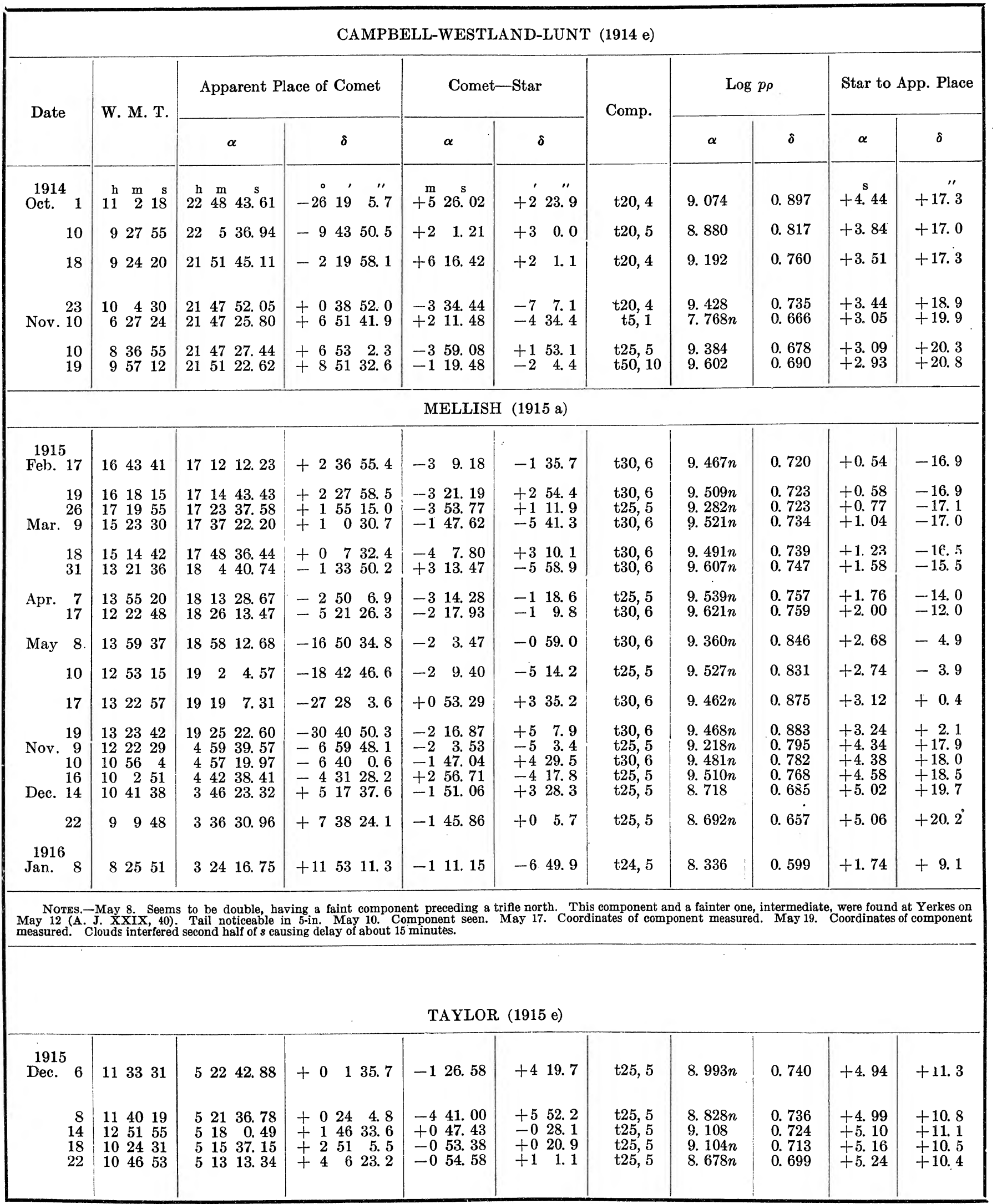




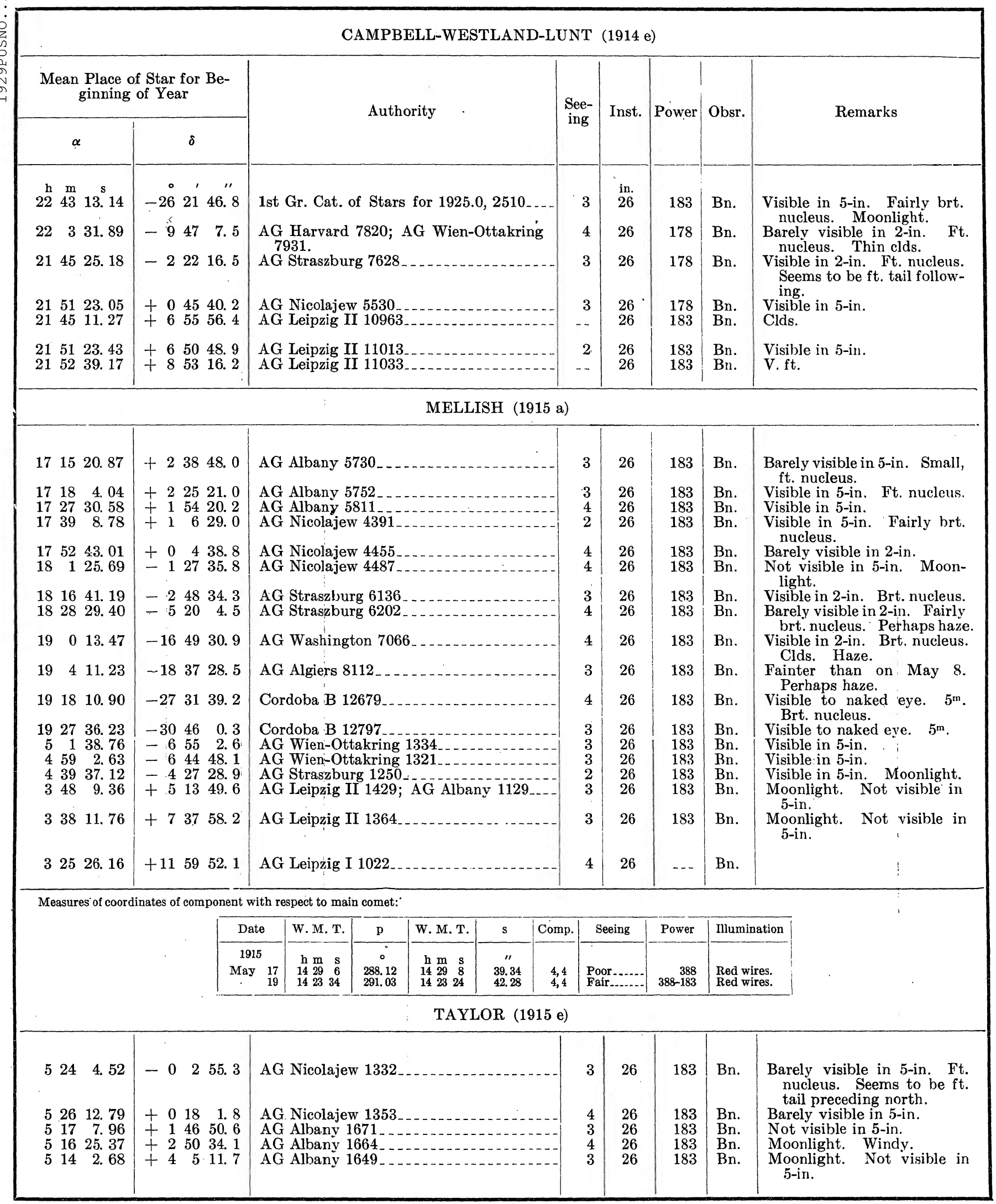




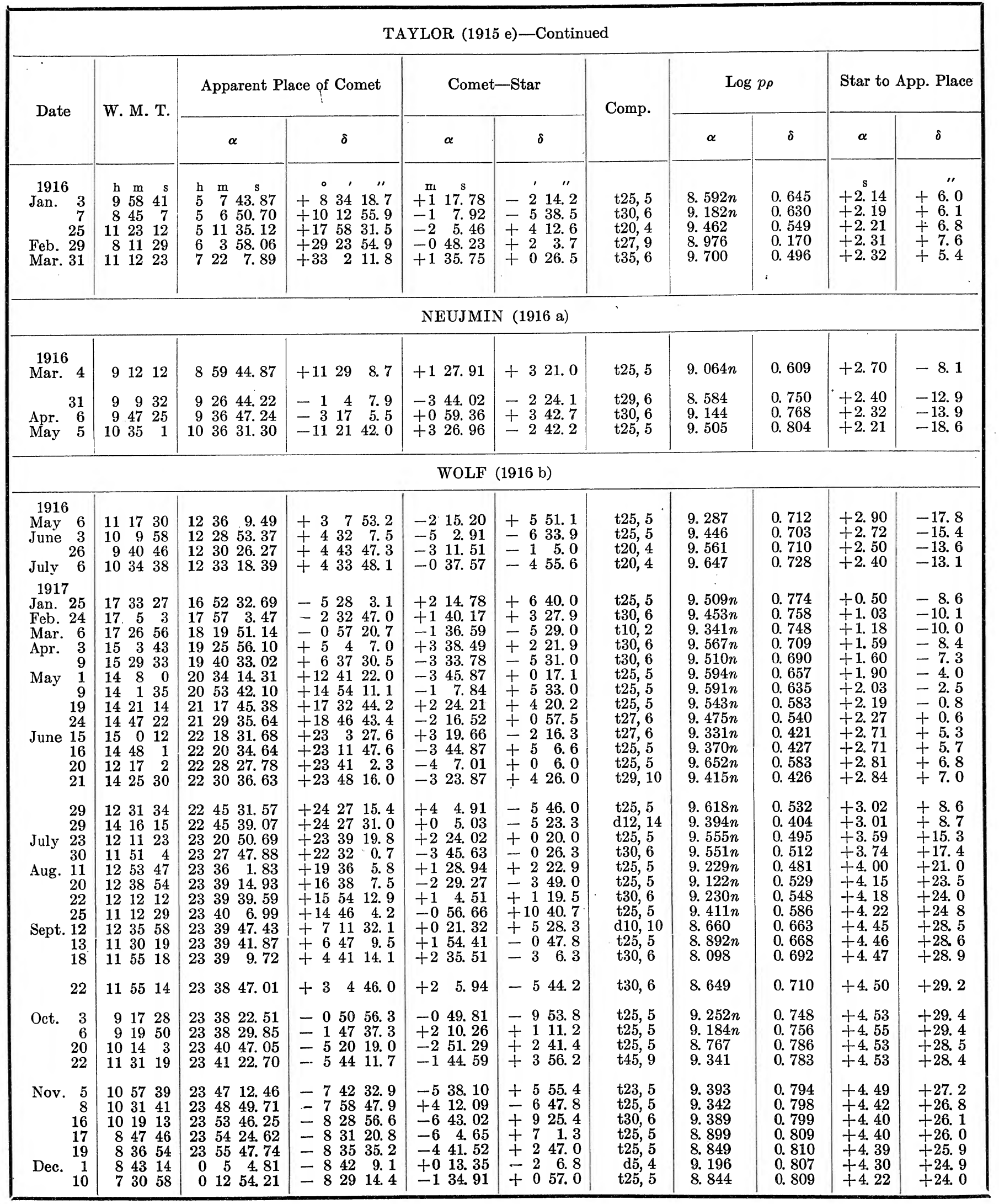


TAYLOR (1915 e)-Continued

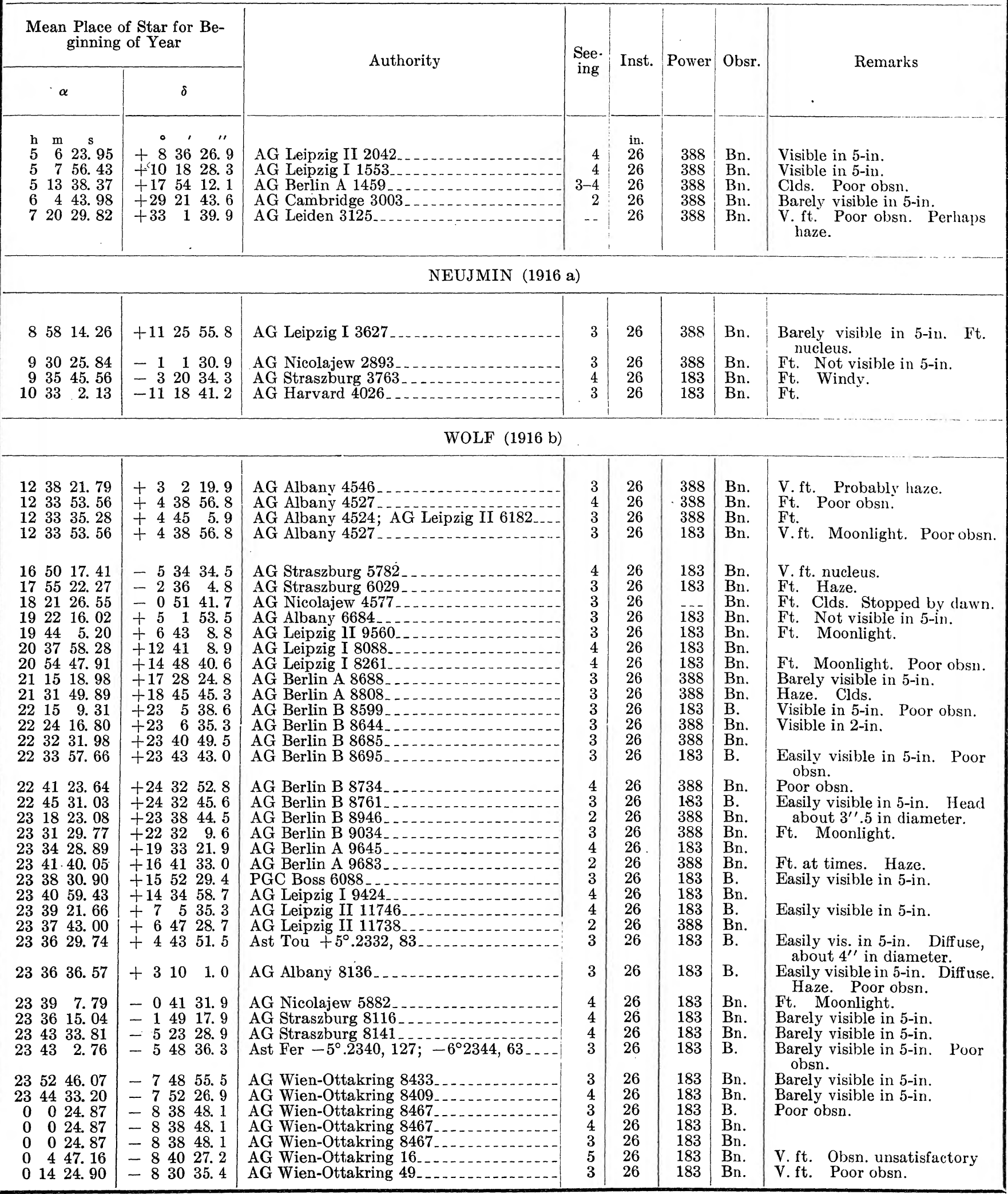

$1757-29-14$ 


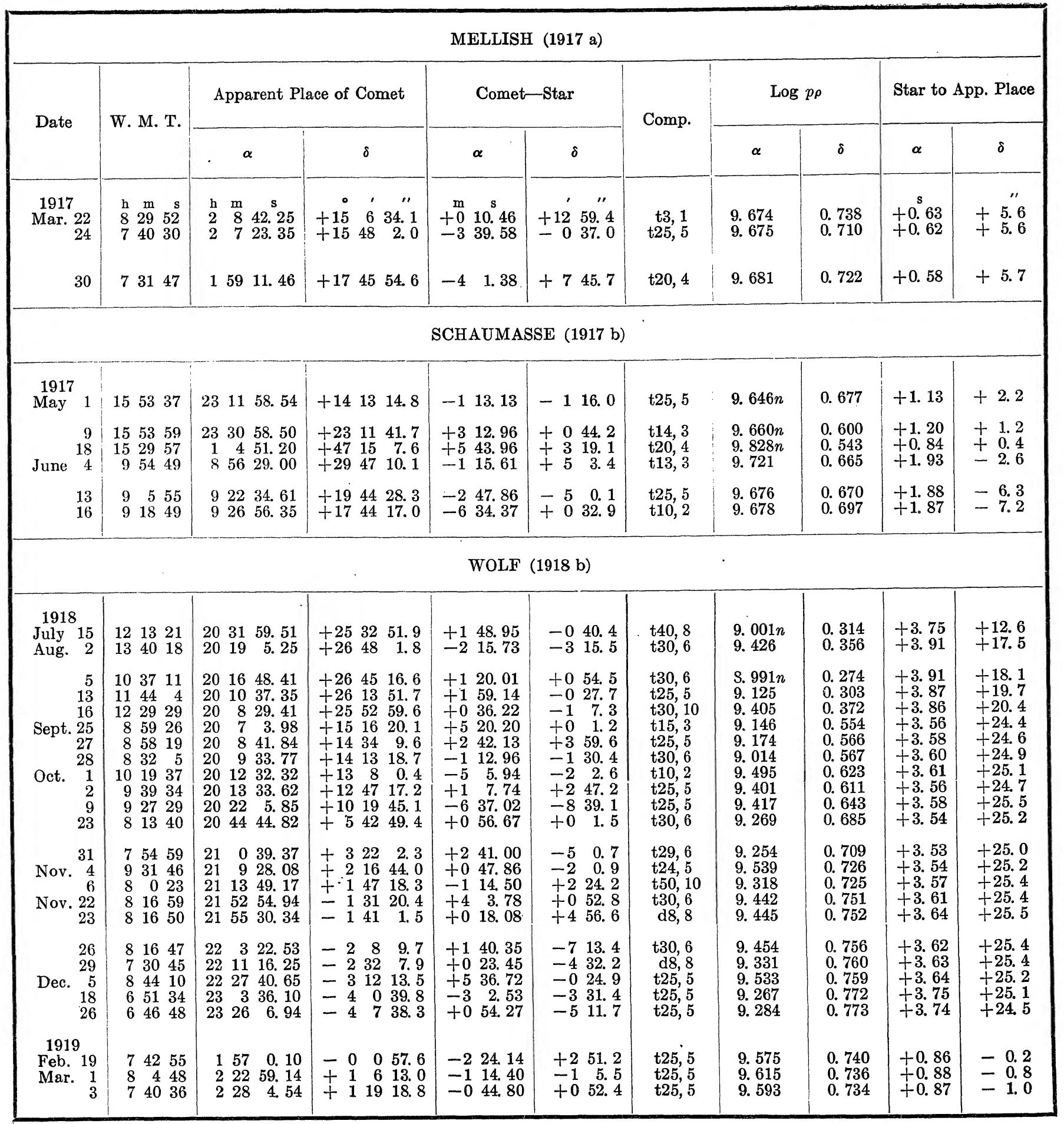




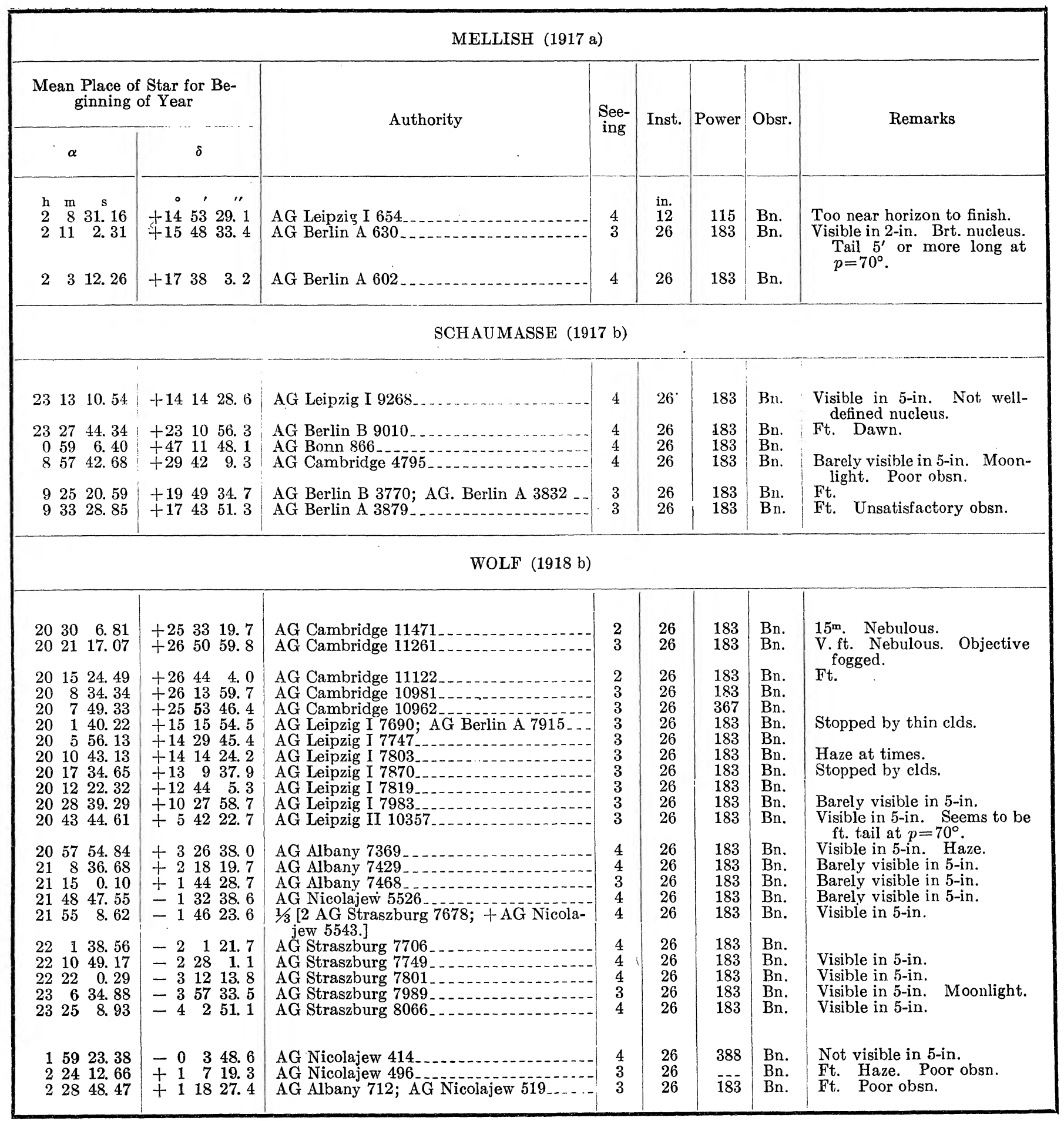




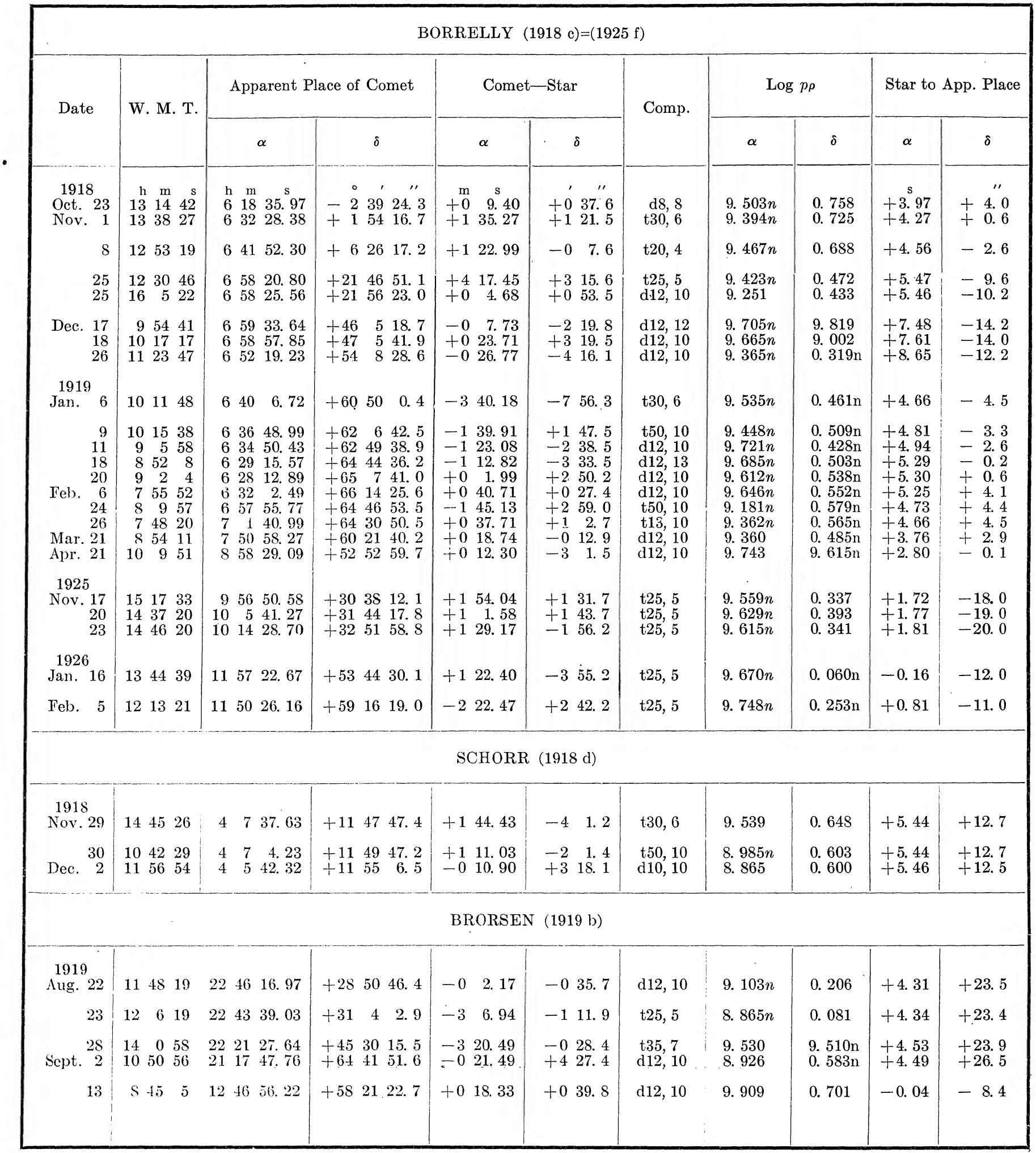




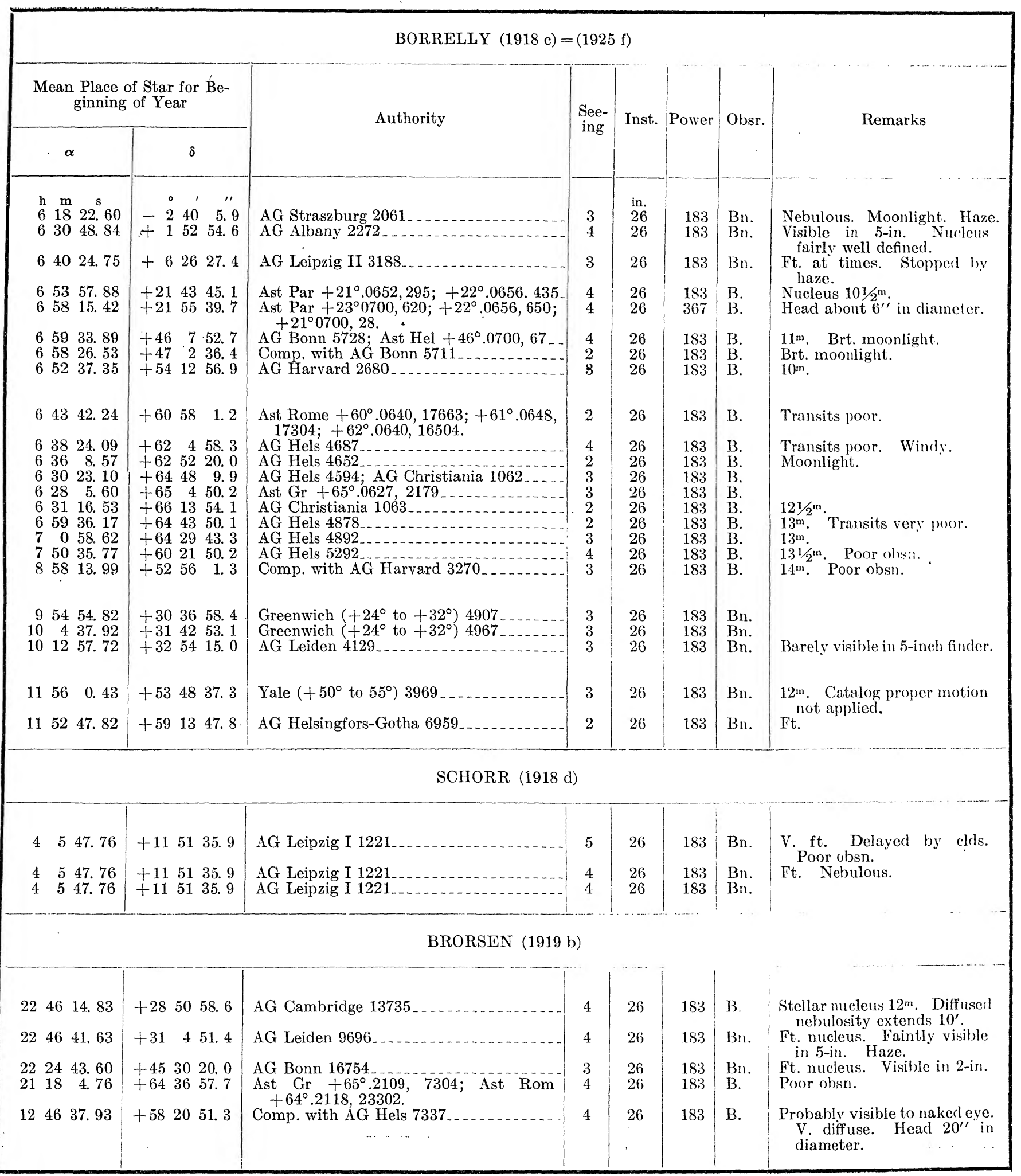




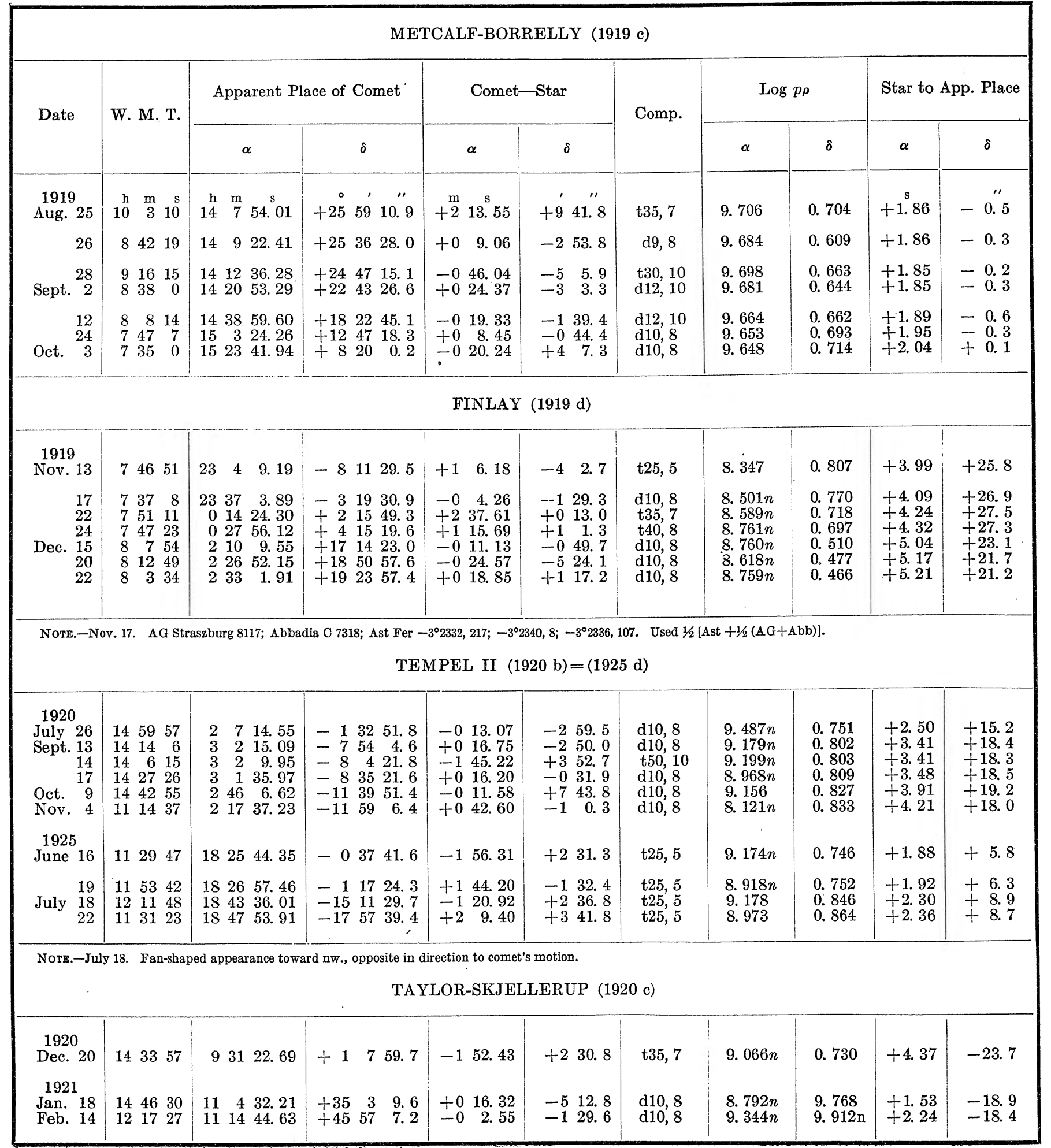




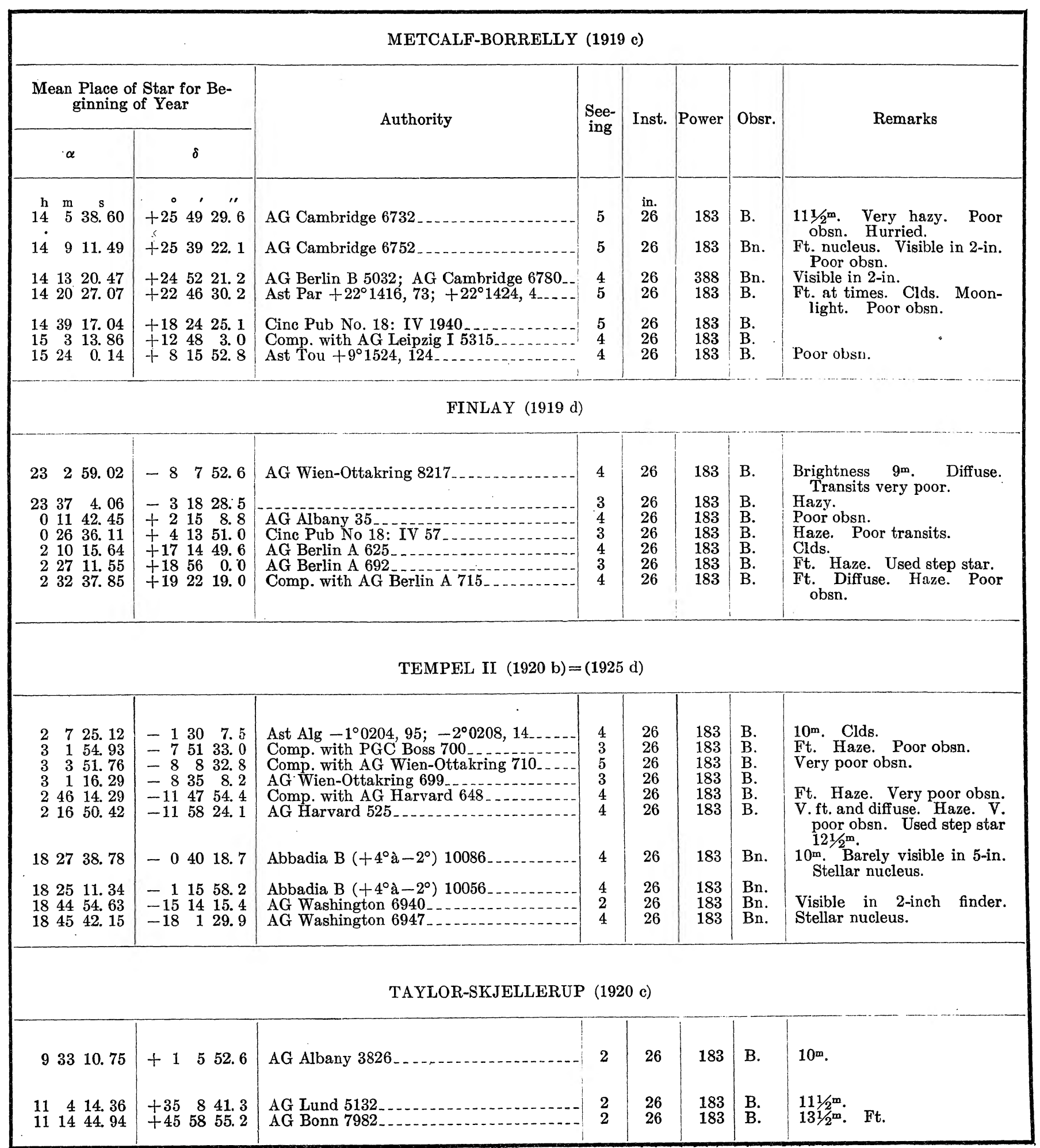




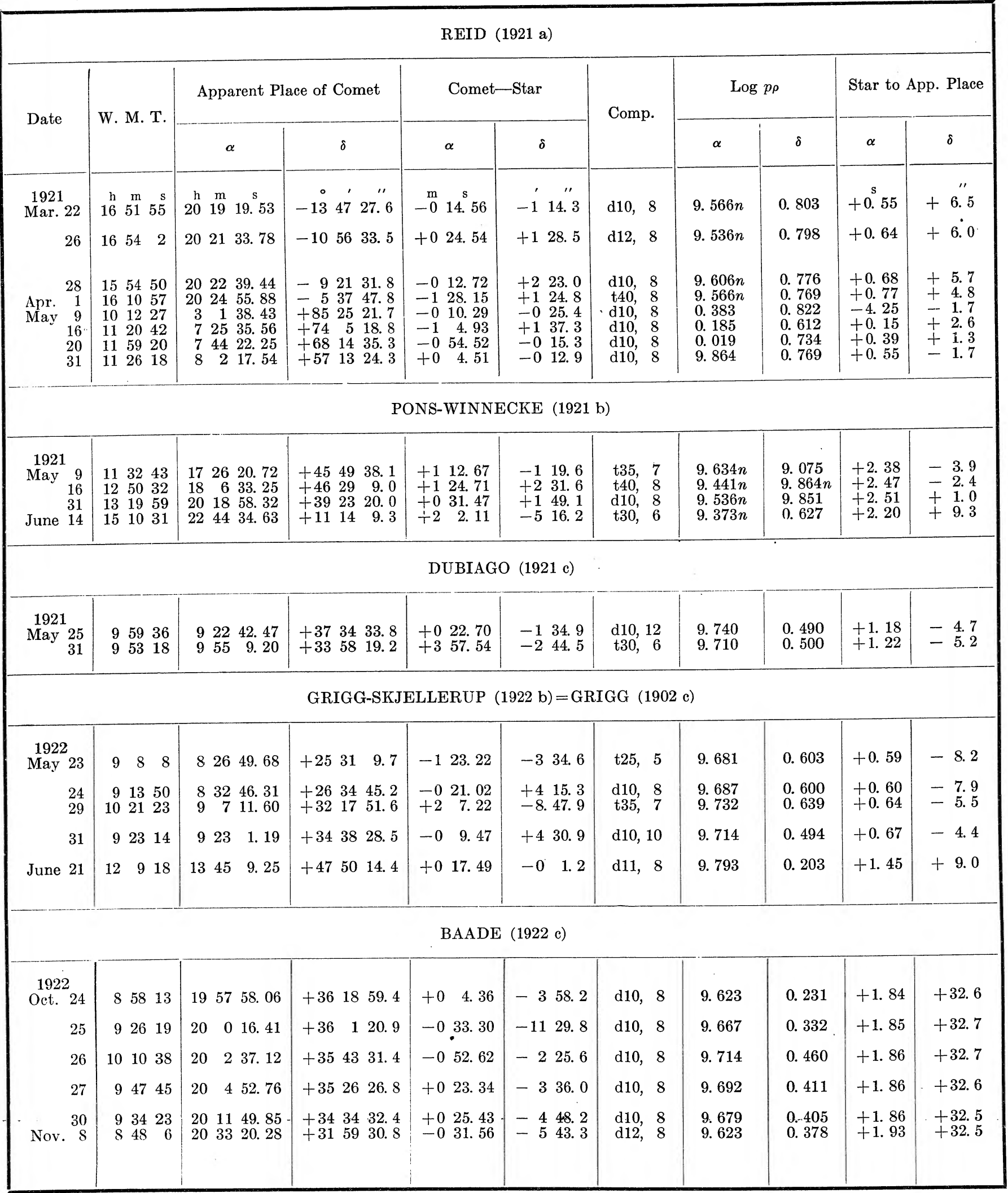




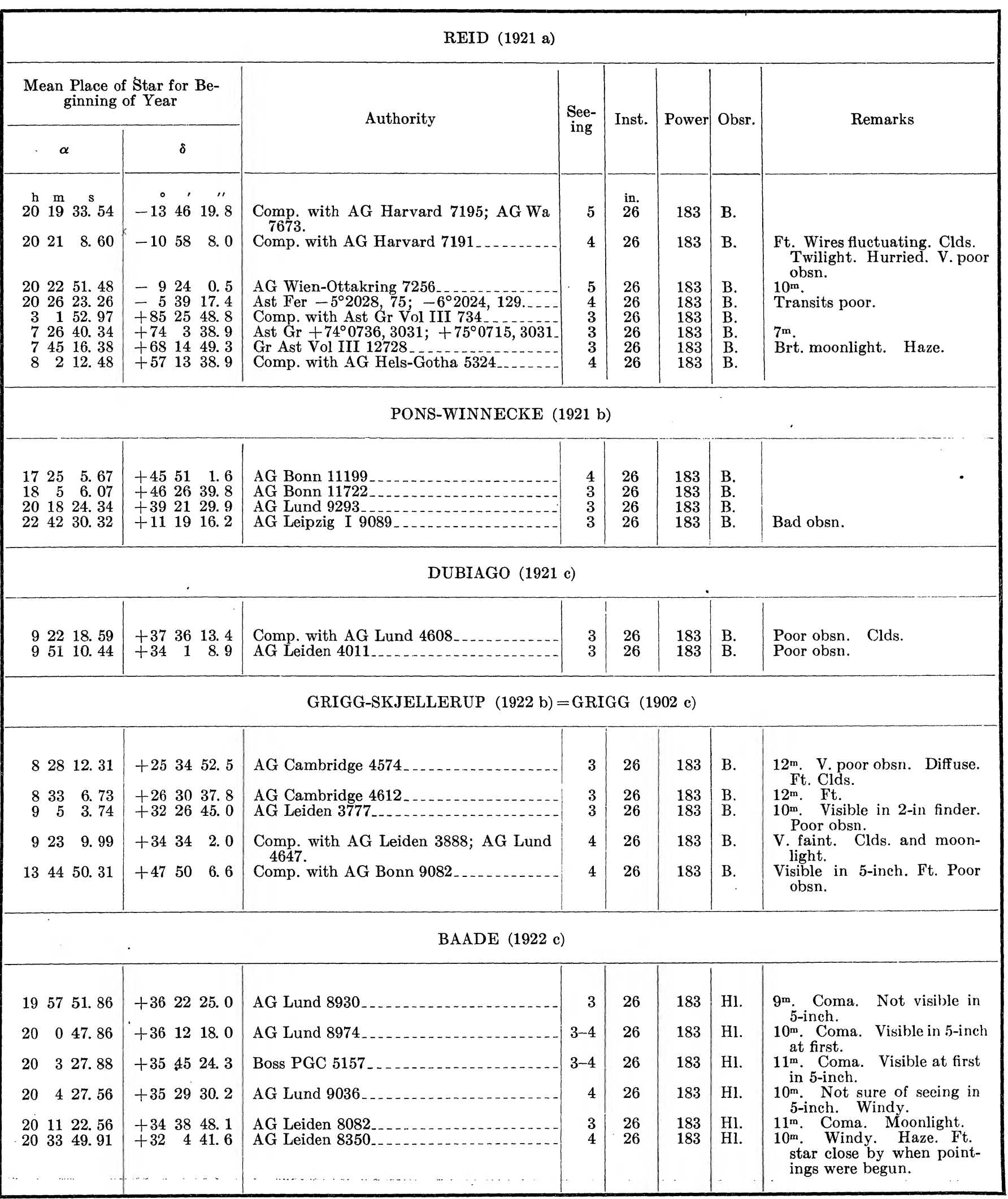


BAADE (1922 c)-Continued

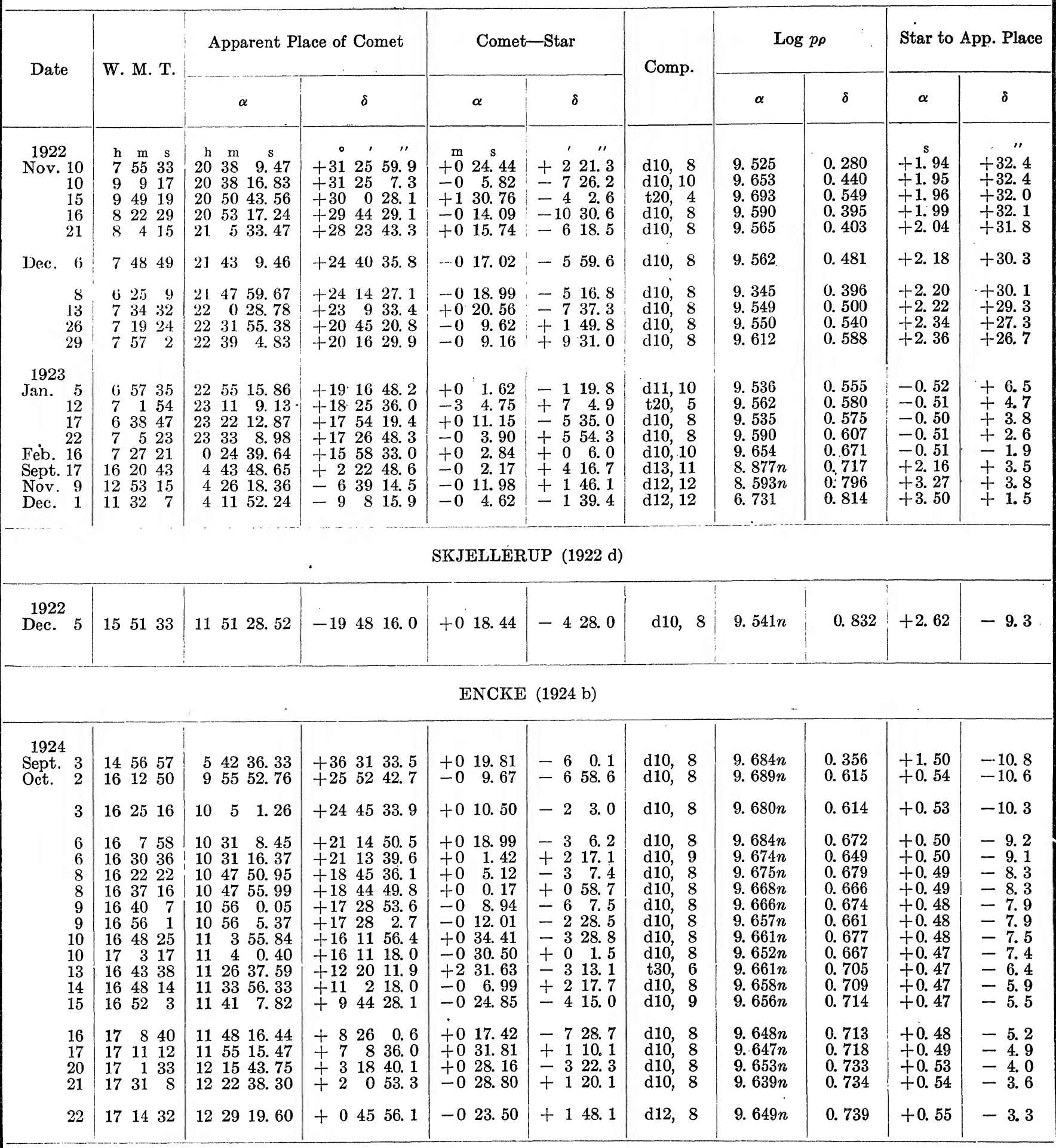

Notes.-Oct. 21. Has 20' tail (hour angl $\theta=-4 \mathrm{~h} 49 \mathrm{~m}$ ) at $\mathrm{p}=303^{\circ}$. 


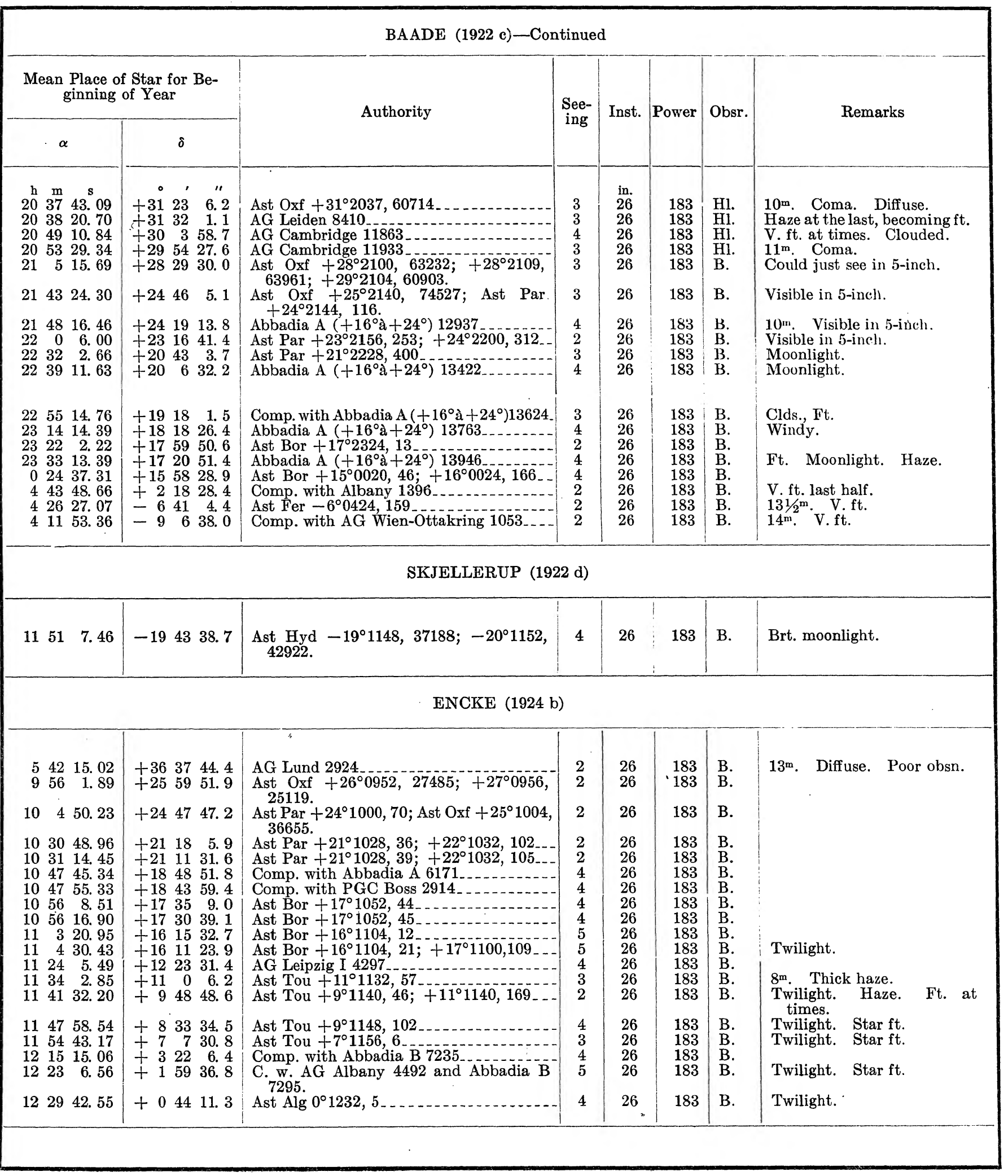




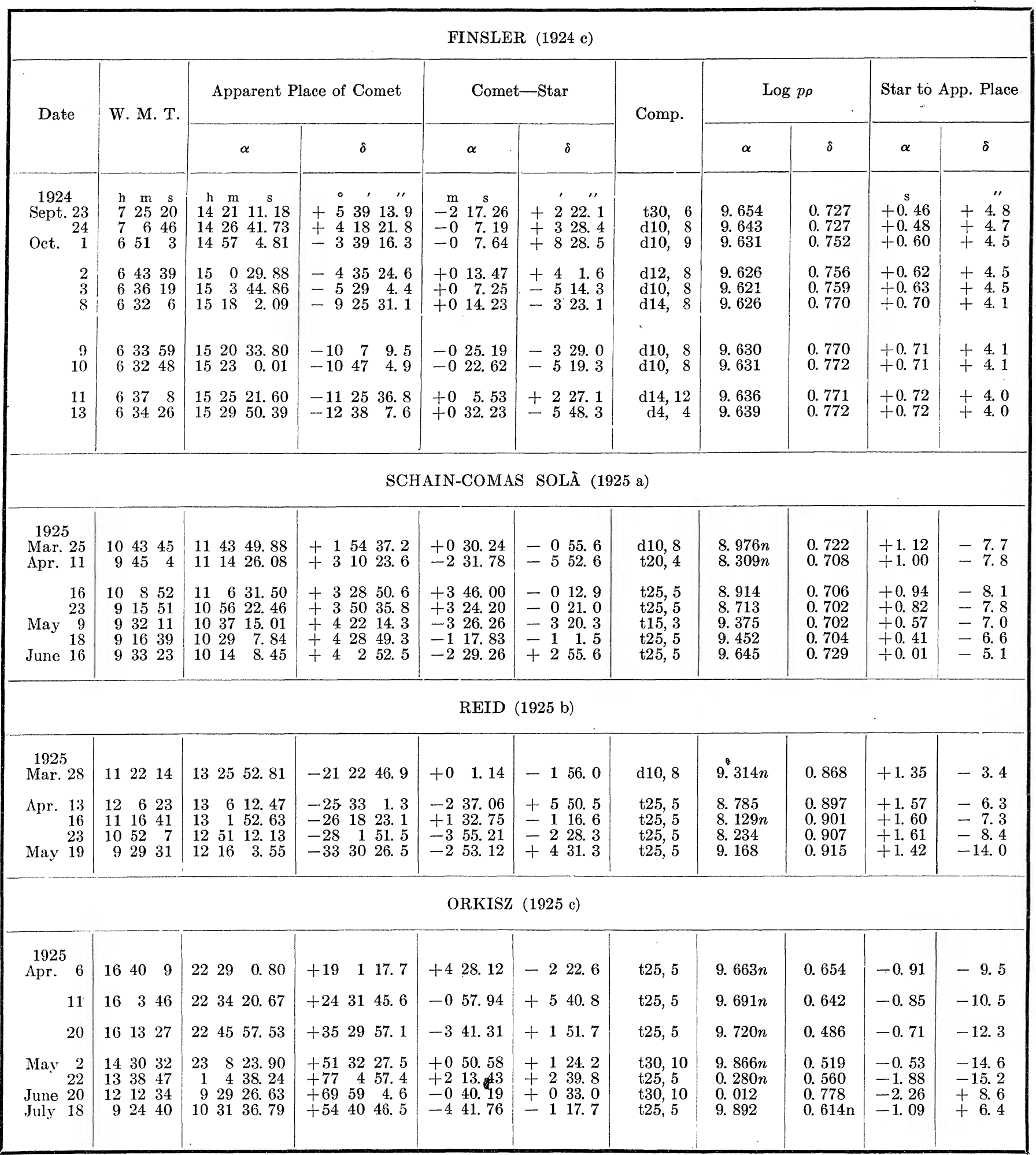




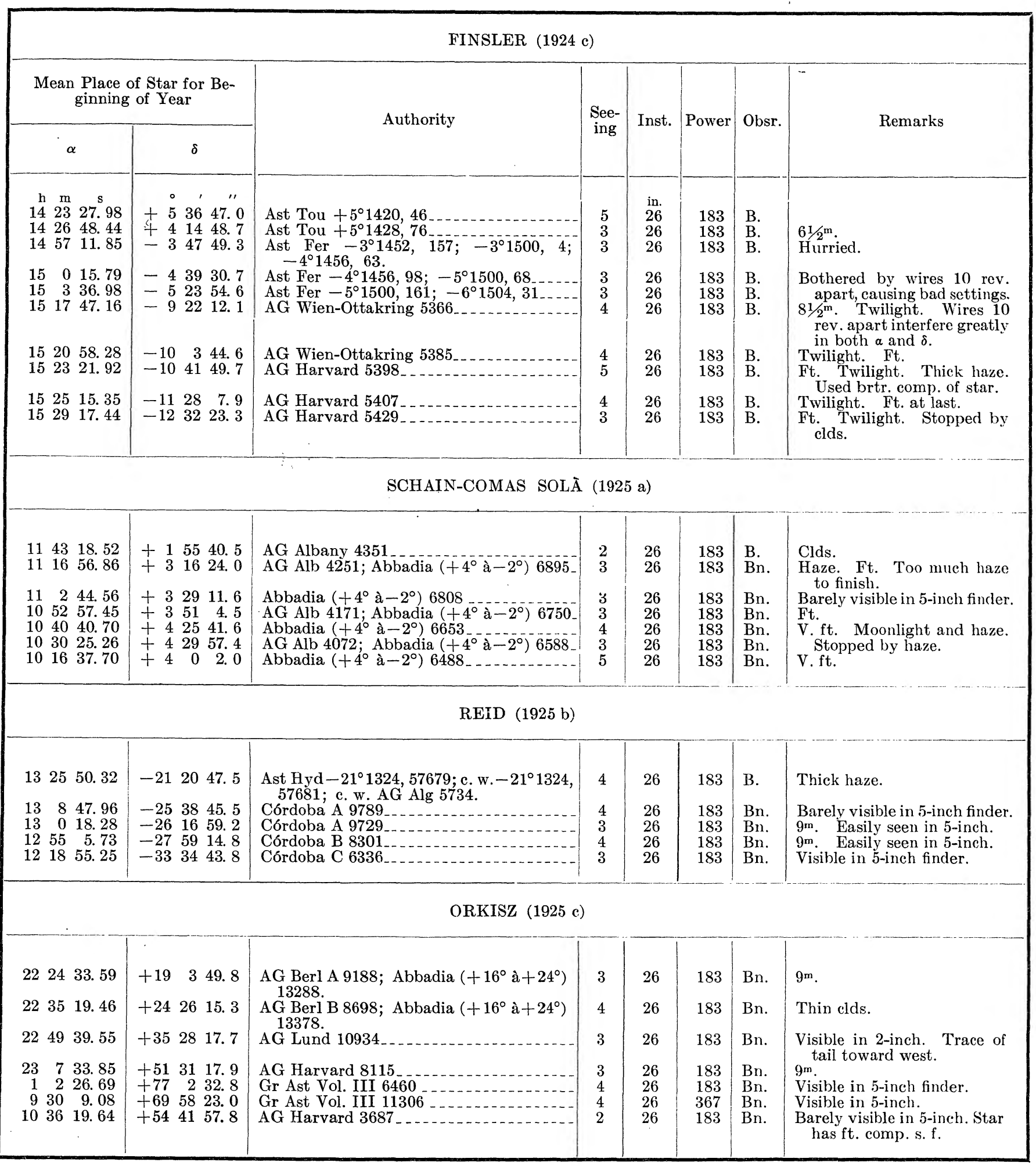




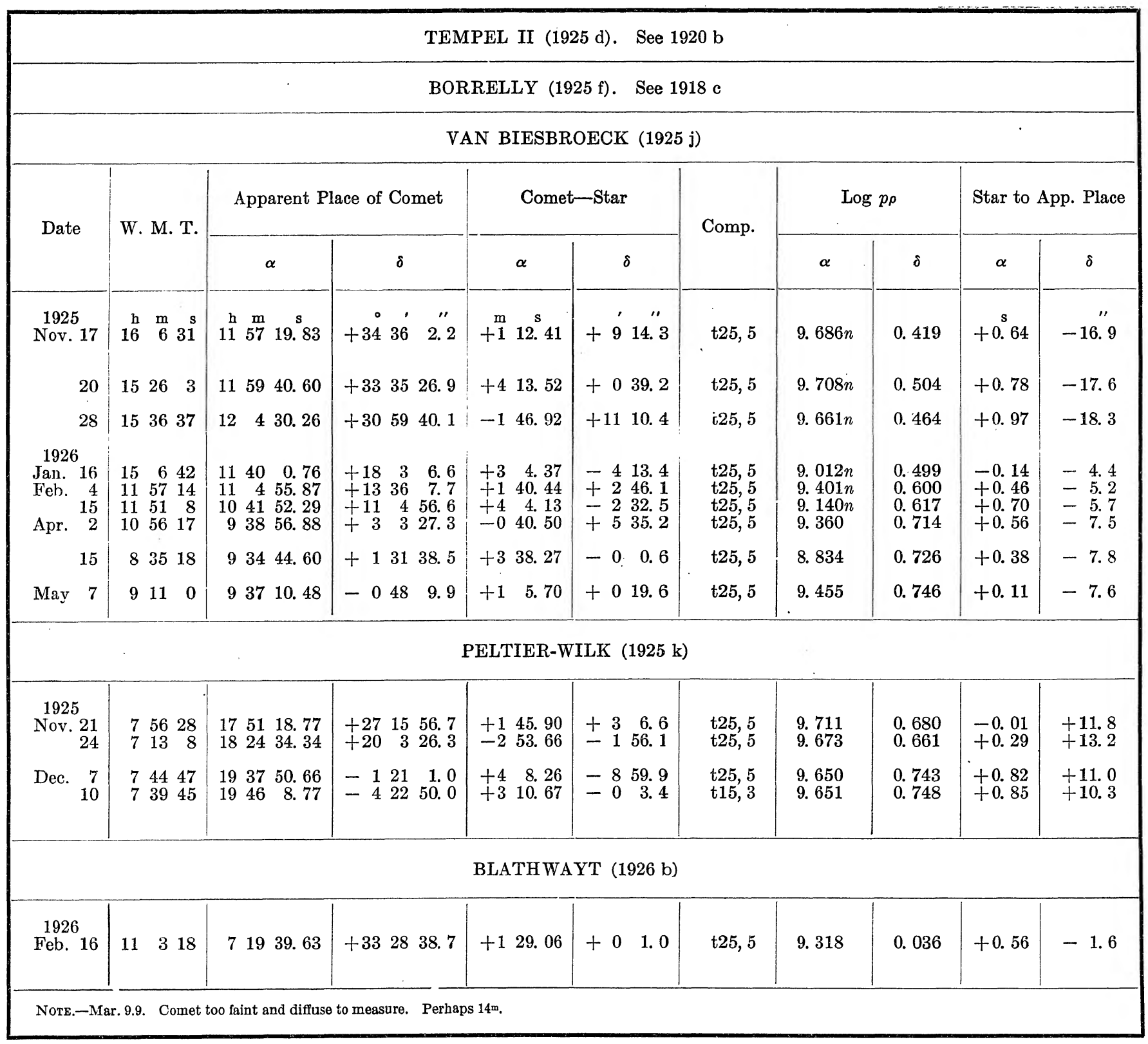


TEMPEL II (1925 d). See $1920 \mathrm{~b}$

BORRELLY (1925 f). See 1918 c

VAN BIESBROECK (1925 j)

\begin{tabular}{|c|c|c|c|c|c|c|c|}
\hline \multicolumn{2}{|c|}{$\begin{array}{l}\text { Mean Place of Star for Be- } \\
\text { ginning of Year }\end{array}$} & \multirow{2}{*}{ Authority } & \multirow{2}{*}{$\begin{array}{l}\text { See- } \\
\text { ing }\end{array}$} & \multirow{2}{*}{ Inst. } & \multirow{2}{*}{ Power } & \multirow{2}{*}{ Obsr. } & \multirow{2}{*}{ Remarks } \\
\hline$\alpha$ & $\delta$ & & & & & & \\
\hline $\begin{array}{rrr}\text { h } & \mathrm{m} & \mathrm{s} \\
11 & 56 & 6.78\end{array}$ & $\begin{array}{r}\circ{ }^{\circ} \prime \prime \\
+34274.8\end{array}$ & AG Leiden 4561 $\ldots$ & 3 & $\begin{array}{l}\text { in. } \\
26\end{array}$ & 183 & Bn. & $\begin{array}{l}9^{\mathrm{m}} \text {. Vis. in 5-inch. Nucleus } \\
\text { well defined. Est. pos. } \\
\text { angle of tail }=320^{\circ} \text {. }\end{array}$ \\
\hline 115526.30 & $+3335 \quad 5.3$ & AG Leiden 4559 & 3 & 26 & 183 & $\mathrm{Bn}$. & Star has ft. comp. n. f. nearly \\
\hline 12616.21 & +304848.0 & $\mathrm{Gr}\left(+24^{\circ}\right.$ to $\left.+32^{\circ}\right) 5653 \ldots$ & 3 & 26 & 183 & Bn. & \\
\hline $\begin{array}{rrr}11 & 36 & 56.53 \\
11 & 3 & 14.97 \\
10 & 37 & 47.46 \\
9 & 39 & 36.82\end{array}$ & $\begin{array}{l}+18724.4 \\
+133326.8 \\
+11734.8 \\
+25759.6\end{array}$ & $\begin{array}{l}\text { Abbadia }\left(+16^{\circ} \grave{a}+24^{\circ}\right) 6469 \\
\text { AG Leipzig I } 4198 \\
\text { Bordeaux }\left(+11^{\circ}\right) 384 \\
\text { Abbadia }\left(+4^{\circ} \grave{a}-2^{\circ}\right) 6215\end{array}$ & $\begin{array}{l}3 \\
4 \\
4 \\
3\end{array}$ & $\begin{array}{l}26 \\
26 \\
26 \\
26\end{array}$ & $\begin{array}{l}183 \\
183 \\
183 \\
183\end{array}$ & $\begin{array}{l}\text { Bn. } \\
\text { Bn. } \\
\text { Bn. } \\
\text { Bn. }\end{array}$ & $\begin{array}{l}\text { Visible in } 5 \text {-inch. } \\
\text { Barely vis. in } 5 \text {-inch. Windy. } \\
\text { Visible in } 5 \text {-inch. } \\
\text { Barely vis. in } 5 \text {-inch. Haze. }\end{array}$ \\
\hline $931 \quad 5.95$ & +13146.9 & Abbadia $\left(+4^{\circ} \grave{a}-2^{\circ}\right) 6155$ & 3 & 26 & 183 & Bn. & $\begin{array}{l}\text { Star has comp. } \\
\text { Barely vis. in 5-inch. Ft. }\end{array}$ \\
\hline $936 \quad 4.66$ & -04821.9 & 1st Gr Cat for $1925.0,1043$ & 3 & 26 & 183 & Bn. & $\begin{array}{l}\text { nucleus. } \\
\text { V. faint. Haze. }\end{array}$ \\
\hline
\end{tabular}

PELTIER-WILK (1925 k)

\begin{tabular}{lll|lll|l}
17 & 49 & 32.88 & +27 & 12 & 38.3 & AG Cambridge 8498
\end{tabular}

$182727.71+20 \quad 5 \quad 9.2$

$193341.58-11212.1$

$194257.25-42256.9$
AG Cambridge 8498

Abbadia $\left(+4^{\circ} \grave{a}-2^{\circ}\right) 10898$

Abbadia $\left(-3^{\circ} \grave{a}-9^{\circ}\right) 6125$

\begin{tabular}{|l|l|l|l|} 
& & & \\
4 & 12 & 115 & $\mathrm{Bn}$. \\
5 & 12 & 115 & $\mathrm{Bn}$. \\
5 & 12 & 115 & $\mathrm{Bn}$. \\
& 12 & 115 & Bn. \\
\hline
\end{tabular}

9m. No well-defined nucleus. $9^{\mathrm{m}}$. Easily visible in 4-inch finder.

Diffuse.

$81 / 2^{\mathrm{m}}$. Too near horizon to finish.

BLATHWAYT (1926 b)

$\begin{array}{lll}7 & 18 & 10.01\end{array}|+332839.3|$ AG Leiden 3105

$2 \mid 26$

Bn. Barely visible in 5-inch. Very ft. nucleus. 